\title{
Colour-emotion associations in individuals with red-green colour blindness
}

\author{
Domicele Jonauskaite ${ }^{\text {Corresp., } 1}{ }^{1}$, Lucia Camenzind ${ }^{1}$, C Alejandro Parraga ${ }^{2}$, Cécile N. Diouf ${ }^{1}$, Mathieu Mercapide \\ Ducommun ${ }^{1}$, Lauriane Müller ${ }^{1}$, Mélanie Norberg ${ }^{1}$, Christine Mohr $^{1}$ \\ 1 Institute of Psychology, University of Lausanne, Lausanne, Vaud, Switzerland \\ 2 Computer Vision Center, Universitat Autònoma de Barcelona, Barcelona, Spain \\ Corresponding Author: Domicele Jonauskaite \\ Email address: domicele.jonauskaite@unil.ch
}

Colours and emotions are associated in languages and traditions. Some of us may convey sadness by saying feeling blue or by wearing black clothes at funerals. The first example is a conceptual experience of colour and the second example is an immediate perceptual experience of colour. To investigate whether one or the other type of experience more strongly drives colour-emotion associations, we tested 64 congenitally red-green colourblind men and 66 non-colour-blind men. All participants associated 12 colours, presented as terms or patches, with 20 emotion concepts, and rated intensities of the associated emotions. We found that colour-blind and non-colour-blind men associated similar emotions with colours, irrespective of whether colours were conveyed via terms $(r=.82)$ or patches $(r=.80)$. The colour-emotion associations and the emotion intensities were not modulated by participants' severity of colour blindness. Hinting at some additional, although minor, role of actual colour perception, the consistencies in associations for colour terms and patches were higher in non-colour-blind than colour-blind men. Together, these results suggest that colour-emotion associations in adults do not require immediate perceptual colour experiences, as conceptual experiences are sufficient. 


\section{Colour-Emotion Associations In Individuals With Red-}

\section{Green Colour Blindness}

Domicele Jonauskaite $^{1 *}$, Lucia Camenzind ${ }^{1}$, C. Alejandro Parraga ${ }^{2}$, Cécile N. Diouf $^{1}$, Mathieu Mercapide Ducommun ${ }^{1}$, Lauriane Müller ${ }^{1}$, Mélanie Norberg ${ }^{1}$, and Christine Mohr ${ }^{1}$

${ }^{1}$ Institute of Psychology, University of Lausanne, Lausanne, Switzerland

${ }^{2}$ Computer Vision Center, Universitat Autònoma de Barcelona, Barcelona, Spain

*Corresponding author:

Domicele Jonauskaite

Institute of Psychology, University of Lausanne, CH-1015, Lausanne, Switzerland

Domicele.Jonauskaite@unil.ch

ORCID: 0000-0002-7513-9766 


\section{Abstract}

2 Colours and emotions are associated in languages and traditions. Some of us may convey

3 sadness by saying feeling blue or by wearing black clothes at funerals. The first example is a

4 conceptual experience of colour and the second example is an immediate perceptual experience

5 of colour. To investigate whether one or the other type of experience more strongly drives

6 colour-emotion associations, we tested 64 congenitally red-green colour-blind men and 66 non-

7 colour-blind men. All participants associated 12 colours, presented as terms or patches, with 20

8 emotion concepts, and rated intensities of the associated emotions. We found that colour-blind

9 and non-colour-blind men associated similar emotions with colours, irrespective of whether

10 colours were conveyed via terms $(r=.82)$ or patches $(r=.80)$. The colour-emotion associations

11 and the emotion intensities were not modulated by participants' severity of colour blindness.

12 Hinting at some additional, although minor, role of actual colour perception, the consistencies in

13 associations for colour terms and patches were higher in non-colour-blind than colour-blind men.

14 Together, these results suggest that colour-emotion associations in adults do not require

15 immediate perceptual colour experiences, as conceptual experiences are sufficient.

16 Word count: 8,255 words (main text only). 


\section{Introduction}

18 We feel blue, see red, and have some black days. As Westerners, we might wear white to

19 weddings and black to funerals. These examples show that colours and affective meanings are

20 associated in natural languages and cultural traditions. Moreover, colour-emotion associations

21 are highly similar across cultures (Adams \& Osgood, 1973; D’Andrade \& Egan, 1974; Gao et

22 al., 2007; Ou et al., 2018; Jonauskaite et al., 2020a). This similarity is indicative of a human

23 psychological universal, which is a mental attribute shared by all or nearly all healthy human

24 beings (see Norenzayan \& Heine, 2005). This universal might be determined by conceptual

25 knowledge, because emotion associations were similar when matched to colour patches or colour

26 words, indicating that immediate colour perception is not necessary for these associations to be

27 reported (Jonauskaite et al., 2020b). To test this possibility, we recruited individuals with

28 congenital red-green colour blindness. Such individuals have never seen colours in the same way

29 as individuals with intact colour vision due to their congenital deficiencies (Linhares, Pinto \&

30 Nascimento, 2008). Yet, colour-blind individuals have been exposed to similar conceptual

31 information, namely similar cultural and linguistic environments as non-colour-blind individuals

32 (Byrne \& Hilbert, 2010). If colour-emotion associations in the two groups are similar,

33 irrespective of whether seeing colour patches or colour terms, we have good reasons concluding

34 that conceptual colour-emotion associations are so well established that sufficient, and that

35 immediate colour perception is not essential for such associations to be reported.

36 We are aware of several older and more recent studies investigating the extent to which colour-

37 emotion associations are shared across cultures (Adams \& Osgood, 1973; D’Andrade \& Egan,

38 1974; Hupka et al., 1997; Madden, Hewett \& Roth, 2000; Gao et al., 2007; Ou et al., 2018; 
39 Jonauskaite et al., 2020a). Some studies reported cross-cultural similarities in, and even claimed

40 universality for, associations between brighter colours and positivity (Specker et al., 2018),

41 associations between colours and affective dimensions (Adams \& Osgood, 1973; Gao et al.,

42 2007; Ou et al., 2018), or colours and emotion terms (D’Andrade \& Egan, 1974). Examples of

43 these associations include red being an active, warm and strong colour, blue, green, and white

44 being positive colours, dark colours being heavy while desaturated colours being passive. Other

45 studies highlighted cross-cultural differences. For instance, envy was associated with black, red,

46 green, yellow, or purple depending on the nation (Hupka et al., 1997). These studies, however,

47 used different methods, usually testing a limited number of colours, emotions, and/or cultures.

48 Recently, Jonauskaite and colleagues (Jonauskaite et al., 2020a) tested 240 colour-emotion

49 associations in 30 nations resulting from associations between 12 colour terms and 20 emotion

50 concepts. For each colour term, participants were free to associate as many emotions as they felt

51 appropriate, in their native language. Results revealed high similarity in the way colours and

52 emotions were associated across nations (average correlation was $r=.88$ ). These cross-cultural

53 results indicate that humans largely share how they associate colours with emotions, at least

54 when colours are presented as terms. Presentation mode does not seem to matter, though, as

55 similar emotions have been associated with colour patches, at least when participants were tested

56 in a Western context. More precicely, in another study, Swiss adults again associated the 12

57 colours with the 20 emotion concepts (Jonauskaite et al., 2020b). One group of participants

58 associated emotions with basic colour terms and the other group with focal colours that best

59 represent these basic colour terms. Both groups chose similar emotions for the same colour

60 concepts, irrespective of whether they were presented as terms or patches (correlation between

61 groups was $r=.82$ ). In a different study, Wang and colleagues (2014) reported high similarity in 
62 term-patch associations for blue but not red. In their study, Chinese participants evaluated red

63 more positively as a term than a patch. Overall, with some potential exceptions, these results

64 suggest that seeing a colour is not key to decide on colour-emotion associations, at least once

65 individuals have reached adulthood.

66 High similarities in colour-emotion associations across cultures and presentation mode do not

67 reveal the mechanisms that drive the formation of shared colour-emotion associations.

68 Considering potential mechanisms, one mechanism might be shared perceptual experiences by

69 most humans (see also, Palmer \& Schloss, 2010; Jonauskaite et al., 2019a). In this case, a direct

70 perceptual experience of colour might lead to an affective experience. For instance, looking at a

71 colour would make one feel a certain emotion or immediately remind of a particular emotion.

72 Another mechanism might be shared conceptual knowledge, accessed and transmitted through

73 language (see Xu et al., 2013, for cultural transmission of colour terms). In that case, colours and

74 emotions would be conceptually associated without necessarily meaning that immediate colour

75 perception itself evokes associations with affective experiences.

76 To test the relative importance of conceptual versus immediate colour experience, we suggest for

77 this study to test colour-emotion associations in populations with colour vision deficiencies. The

78 most frequent colour vision deficiency is congenital red-green colour blindness. Here, affected

79 individuals can discern a smaller number of colours than individuals with complete colour vision

80 (Neitz \& Neitz, 2000; Linhares, Pinto \& Nascimento, 2008). Red-green colour blindness, also

81 called Daltonism after John Dalton (Dalton, 1798), affects around 8\% of the male population and

82 around $0.6 \%$ of the female population of European-Caucasian origin (Sharpe et al., 1999; Birch,

83 2012). Such individuals confuse certain colours along the red-green axis (e.g., red and brown, 
84 green and brown, pink and grey, grey and green, etc.; Moreira et al., 2014) and likely see the 85 world in bluish-yellowish colours (Judd, 1949; Byrne \& Hilbert, 2010). Individuals with red86 green colour blindness have never seen certain colours the way individuals with intact colour 87 vision do, but have been exposed to their shared cultural and linguistic environments (e.g., traffic colours; Almustanyir \& Hovis, 2020). Accordingly, if individuals with and without red-green colour blindness display similar colour-emotion associations, we can argue that shared conceptual knowledge is sufficient for colour-emotion associations to occur.

91 Studies assessing colour naming and colour arrangements support the importance of conceptual

92 knowledge. In case of colour naming, colour-blind individuals were able to name colours

93 indicating that they learned to differentiate colours, irrespective of whether they look the same or

94 different to colours perceived by individuals with intact colour vision (Jameson \& Hurvich, 95 1978; Paramei, 1996; Bonnardel, 2006; Nagy et al., 2014; Moreira et al., 2014). Bonnardel 96 (2006) found that consensus in colour naming ranged between $52 \%$ and $74 \%$ for colour-blind 97 and non-colour-blind individuals. The highest consensus emerged when participants had to name 98 colour chips using one of eight colour terms (i.e., constrained colour naming task; $74 \%$ 99 consensus). Some of the chips were focal colours (i.e., the best examples of each colour 100 category) while others were not. The lowest consensus emerged for a task that least involved 101 language (i.e., freely grouping colour chips into colour categories, $52 \%$ consensus). For colour 102 arrangements, colour-blind individuals mentally arranged colours more similarly to non-colour-

103 blind individuals when colours were presented as terms than as patches (Shepard \& Cooper, 104 1992; Saysani, Corballis \& Corballis, 2018a). When presented with terms, colour-blind 105 individuals used three colour axes (i.e., red-green, blue-yellow, and dark-light) to arrange 106 colours. When presented with patches of focal colours, colour-blind individuals collapsed 
107 colours along the red-green axis and used only two axes to arrange colours. Taken together,

108 conceptual knowledge seems essential for colour naming and colour arrangements. Nonetheless,

109 colour terms and colour patches might be treated somewhat differently by colour-blind

110 individuals. If so, colour-blind individuals might also treat colour-emotion associations

111 differently when actually reading a colour term or seeing a colour patch.

112 To test the importance of conceptual knowledge and immediate perceptual colour experience, we

113 assessed 240 colour-emotion associations in individuals with and without red-green colour

114 blindness using a previously established methodology (Jonauskaite et al., 2019c, 2020b,a). Part

115 of each group associated emotion terms with colour terms while the remainder associated

116 emotion terms with colour patches displaying focal colours. Emotion terms were presented in a

117 circular format (Scherer, 2005; Scherer et al., 2013). We compared colour-emotion associations

118 between colour-blind and non-colour-blind individuals as well as between colour terms and

119 colour patches in each group. If shared conceptual knowledge is sufficient for colour-emotion

120 associations to occur, we would expect high similarities in colour-emotion associations between

121 individuals with and without colour blindness. We would also expect high similarity in colour-

122 emotion associations between colour terms and colour patches in colour-blind individuals. If,

123 however, previous or immediate perceptual colour experiences are necessary for consistent

124 colour-emotion associations to occur, we would expect differences in colour-emotion

125 associations between individuals with and without colour blindness (e.g., see Álvaro et al., 2015,

126 for colour preferences). These differences should be more pronounced when actual perceptual

127 colours (i.e., colour patches) rather than colour terms are evaluated, since perceptual colours

128 appear differently to individuals with and without colour blindness (Byrne \& Hilbert, 2010). We 
129 would also expect lower consistency between colour terms and colour patches in colour-blind

130 individuals.

131 In addition to comparing colour-blind and non-colour-blind individuals, we further modelled

132 colour blindness as a continuum. We tested whether the strength of colour blindness predicted

133 colour-emotion associations. We chose to treat colour blindness as a continuum due to variations

134 in physiological and behavioural expressions of colour blindness. Red-green colour blindness

135 results from changes in the photopigments in the cone receptors coding for long ("reddish"; L-

136 cones) or medium (“greenish”; M-cones) wavelengths (Parry, 2015). For some individuals, cones

137 are completely missing (dichromatic vision), while for others, they are malfunctioning

138 (anomalous trichromatic vision). The degree of perceptual confusion is related to the degree of

139 individuals' physiological impairments (Neitz \& Neitz, 2000). Many previous studies considered

140 only individuals with dichromatic vision (Jameson \& Hurvich, 1978; Shepard \& Cooper, 1992;

141 Paramei, Bimler \& Cavonius, 1998; Moreira et al., 2014; Saysani, Corballis \& Corballis, 2018a).

142 However, such individuals comprise just $28.5 \%$ of all colour-blind men of European origin (i.e.,

$1432.3 \%$ of the general population of European males; Sharpe et al., 1999). Thus, we decided to

144 freely sample from the colour-blind population and include both individuals with dichromatic

145 vision and anomalous trichromatic vision (similar to, Paramei, 1996; Bonnardel, 2006; Nagy et

146 al., 2014). 


\subsection{Participants}

149 We recruited 130 men, 64 were colour-blind by self-report, which was confirmed with colour

150 vision tests (see Colour Vision Tests for further details). About half of the participants took part

151 in the colour terms condition (associating terms with emotions, Table 1) and the other half took

152 part in the colour patches condition (associating patches with emotions, Table 1). All participants

153 lived in Switzerland. Most participants were either students or staff members of a local

154 university. They were fluent French speakers, apart from one participant who was excluded from

155 the analyses (see Table 1 ). Age did not differ between study groups, $F(3,125)=1.50, p=.218$.

[Insert Table 1 around here]

159 Based on a related previous publication, where we ran a 2 x 12 mixed-design MANOVA to

160 compare emotion associations between terms and patches (Jonauskaite et al., 2020b), we

161 expected a large effect size $(V=.55)$. We entered this effect size in the $\mathrm{G}^{*}$ Power sample size

162 calculator (Faul et al., 2007) together with the expected alpha (.05) and beta (.80) levels. We

163 obtained 26 participants as a minimal total sample size. Yet, we decided to collect more

164 participants to be able to also consider a variability in the expression of colour blindness as well

165 as exclude weak colour-blind participants from part of the analyses. 
166 Participation was voluntary and remunerated with monetary reward (CHF 20 in gift vouchers).

167 The study was conducted in accordance with the principles expressed in the Declaration of

168 Helsinki (World Medical Association, 2013). We received ethics approval from the Research

169 Ethics Commission of the University of Lausanne (C_SSP_032020_00003).

\subsection{Colour Stimuli}

171 We used red, orange, yellow, green, turquoise, blue, purple, pink, brown, white, grey, and black

172 as colour stimuli. Eleven of these colour stimuli represent the principal colour categories

173 (Biggam, 2012). We also included turquoise because it covers the blue-green range. In the terms

174 condition, colour stimuli were presented as French colour terms written in black ink (Spence,

175 1989, see Table S 1). In the patches condition, colour stimuli were presented as colour patches.

176 Colour patches displayed the best exemplars of each colour category (i.e., focal colours, Table 2,

177 Lindsey \& Brown, 2014), and have been used in native French speakers in Switzerland

178 (Jonauskaite et al., 2020b).

179

180

[Insert Table 2 around here]

181

182

\subsection{Emotion Assessment}

183 We used the Geneva Emotion Wheel (GEW 3.0; Figure 1; Scherer, 2005; Scherer et al., 2013) to

184 measure emotion associations with colours. GEW is a validated self-report measure of the

185 feeling component of emotion. Twenty emotion concepts are represented along the 
186 circumference of a wheel. These emotion concepts are organized along two axes. The horizontal

187 axis represents valence, also known as evaluation or pleasantness (positive vs. negative). The

188 vertical axis represents power, also known as control, dominance, or potency (strong vs. weak).

189 Emotion concepts can further be categorised in terms of arousal, also known as activation (high

190 arousal vs. low arousal), based on complementary research studies (Fontaine, 2013; Soriano et

191 al., 2013). We reported this categorisation in a previous related study (Jonauskaite et al., 2020b)

192 and here in Table S 2. Circles of increasing size connect the centre of the wheel with the

193 circumference of the wheel. These circles denote five degrees of emotion intensity, coded from 1

194 (smallest circle; weakest intensity) to 5 (biggest circle; strongest intensity), or 0 if no emotion is

195 chosen (little square). The Swiss Centre for Affective Sciences provides the validated French

196 version of the GEW (Table S 1.).

[Insert Figure 1 around here]

\subsection{Colour Vision Tests}

201 Red-green colour blindness varies in severity. This variation can be behaviourally captured with 202 colour vision tests. In this study, we used the Ishihara test (Ishihara, 2000), the Farnsworth test

203 (Farnsworth, 1947), and the Lanthony test (Lanthony, 1978a,b). Detailed information regarding

204 testing and scoring of the three behavioural tests appears in Supplementary material. These and 205 other similar behavioural tests do not seem to rely on higher cognitive functions. Rather, they 206 rely on the discrimination of primary visual features, since they have been successfully used to 
207 assess colour vision in other animal species (e.g., dogs, seals; Scholtyssek, Kelber \& Dehnhardt, 208 2014; Siniscalchi et al., 2017).

\subsection{Procedure}

210 We performed the colour terms and colour patches conditions as similarly as possible, but had to

211 also account for the different study material. The procedure was identical to a previous study

212 (Jonauskaite et al., 2020b). Below, we detail what was comparable for conditions (see Common

213 to Both Conditions), followed by the description of the terms condition procedure and the

214 patches condition procedure.

\subsubsection{Common to Both Conditions}

216 Upon arrival to the welcome room, we gave participants relevant study information. Those who

217 agreed to participate signed the written informed consent form (see Figure 2 for procedure).

218 Next, we tested participants' colour vision with the Ishihara test. All colour vision tests were

219 conducted as physical tests under the same conditions of artificial office light. Afterwards,

220 participants were invited to the testing room. The computer monitor was the only source of

221 illumination in the testing room. All participants performed the experiment on the same monitor:

222 Eizo ColourEdge CG247 24.1" (inches) LCD display, with an in-built self-calibration sensor. We

223 set the temperature of the monitors to $6500 \mathrm{~K}$, gamma: 2.2 , contrast: $100 \%$, and brightness:

$224120 \mathrm{~cd} / \mathrm{m}^{2}$. Resolution was 1920 x 1200 pixels and the frame rate was $59.90 \mathrm{~Hz}$. The eye-screen

225 distance was approximately $70 \mathrm{~cm}$.

226 Participants completed either the terms or the patches condition. Experimenters were available

227 for questions at any point during the experiments. After the main experiment, participants 
228 returned to the welcome room and completed the Farnsworth D-15 and Lanthony D-15 tests.

229 These tests were given in a randomised order across participants. Once participants completed

230 the first test, the completed test was hidden and they were asked to complete the second test.

231 Upon the completion of both tests, participants were debriefed and remunerated. Participants

232 were invited to ask questions and received a debriefing sheet with written information and

233 contact details for future references. The entire experiment took between 50 and 70 minutes.

\subsubsection{Colour Terms Condition}

235 The colour terms condition was performed in the laboratory testing room. We used an existing

236 online survey link (https://www2.unil.ch/onlinepsylab/colour/main.php); also used to collect data

237 remotely for a larger ongoing International Colour-Emotion Survey online (Mohr et al., 2018;

238 Jonauskaite et al., 2020a). In the current experiment, participants accessed the online survey on

239 our laboratory computer to ensure comparability between the two experimental conditions.

240 The survey started with an information page. On the next pages, the task was explained, namely

241 to associate colour terms with emotion concepts, presented on the GEW (see Emotion

242 Assessment). Participants had to perform a manipulation check exercise to make sure they

243 understood the task. In particular, participants had to correct the responses of an imaginary

244 person (Peter). In the following experimental part, participants saw the 12 colour terms written in

245 black ink on a grey background, presented sequentially and in random order above the GEW (see

246 Colour stimuli and Table 2). Participants were asked to choose one, several, or none of the GEW

247 emotion concepts that they associated with each colour term. They also rated intensities of each

248 associated emotion by choosing circles of different sizes, which were later coded as 1-5 ratings. 
249 After the colour-emotion association task, participants provided demographic information and

250 saw results from a previous related marketing experiment in graphic format.

\subsubsection{Colour Patches Condition}

252 We performed the colour patches condition in the same laboratory testing room as the terms

253 condition. The experiment started with an information page explaining the task, namely to

254 associate colour patches with emotion concepts, presented on the GEW (see Emotion

255 Assessment). Participants proceeded to the next page if they understood the task. Then, three

256 example colours followed. For the examples as well as for the main task, participants were

257 presented with a colour patch $\left(15^{\circ} \times 15^{\circ}\right.$ subtended angle) on a neutral grey background (see

258 Table 2). They were instructed to focus on the colour patch. Participants chose when to move to

259 the subsequent page but no earlier than 5 seconds after it appeared on the screen. On each

260 subsequent page, in analogy to the terms condition, participants associated one, several, or none

261 of the GEW concepts with the target colour patch and rated the intensity of each associated

262 emotion concept. While associating emotions, participants could see the target colour on the

263 small GEW squares as well as on the chosen intensity circles (Figure 2.B Experiment 2). There

264 were 12 experimental colour patches presented in randomised order (see Colour stimuli and

265 Table 2). Colour values were adapted for the monitor (see Apparatus in Supplementary

266 material). We collected these data in the laboratory to ensure accurate colour presentation.

267 After the colour-emotion association task, participants completed the colour-naming task with

268 the same colour patches. Each colour patch was presented 12 times in randomised order and

269 paired with one of the colour terms (total of 144 presentations). Participants had to evaluate how

270 likely they would be using this colour term to name a particular colour patch from "not at all" 
271 (converted to 0) to "very likely" (converted to 100). For example, participants would see a green

272 colour patch and have to respond how likely they would be to call it purple. Not all participants

273 in the patches condition performed the colour-naming task (22 colour-blind and 33 non-colour-

274 blind completed the task). We decided to add this task after the first 10 colour-blind participants

275 had been tested. After these two tasks, participants provided demographic information,

276 analogous to the terms condition, on a paper questionnaire.

277

278

[Insert Figure 2 around here]

279

280

\subsection{Data Preparation}

281 The raw data can be accessed following this link: https://forsbase.unil.ch/project/study-public-

282 overview/16969/0/. We cleaned the data based on colour blindness scores by creating the Colour

283 Blindness Index.

\subsubsection{Colour Blindness Index}

285 We used errors on the colour blindness tests to create a single measure of colour blindness - the

286 Colour Blindness Index. This index served a dual purpose. First, we could ensure accurate

287 participant re-categorisation into colour-blind and non-colour-blind participants. Second, we 288 obtained a continuous measure of colour blindness. 
289 To determine the colour blindness indices, we used a principal component analysis on the

290 correlation matrix of the number of errors on the Ishihara test, the number of crossing errors on

291 both the Farnsworth D-15 and Lanthony D-15 tests, and the number of neighbour errors on both

292 the Farnsworth D-15 and Lanthony D-15 tests (see Supplementary material for scoring). The

293 principal component analysis resulted in two factors with Eigenvalues greater than 1 (i.e., 2.79

294 and 1.07 respectively for factors 1 and 2). The first factor explained $55.7 \%$ of the variance and

295 the second factor explained an additional $21.3 \%$ of the variance. The first factor separated the

296 colour-blind participants from the non-colour-blind participants, and we called this factor the

297 colour blindness index (see Figure S 1A). The second factor was difficult to interpret and did not

298 separate participants by colour blindness (see Figure S 1B). Thus, we disregarded it. In Table S

299 3, we present the loadings of each item for both factors.

300 The visual inspection of the frequency distribution of the colour blindness index (Figure S 1A)

301 indicates that it might consist of three different distributions. The most leftward distribution $(<-$

302 0.6) included only non-colour-blind participants plus one colour-blind participant by self-report.

303 Thus, the latter participant was most likely not colour-blind; he passed both the Farnsworth D-15

304 and Lanthony D-15 tests, and was categorised as "unsure" on the Ishihara test. The most

305 rightward distribution ( $>0.2)$ included only colour-blind participants by self-report, thus, these

306 participants had relatively strong colour blindness. The intermediate distribution (between -0.6

307 and 0.2) included both self-reported colour-blind and non-colour-blind participants. Participants

308 with these scores might have (very) weak colour blindness or no colour vision impairment but

309 nevertheless made errors for other reasons (e.g., inattentiveness). 
310 For the group-level analyses (see below), we considered only the two extreme groups (i.e., re-

311 categorised non-colour-blind and re-categorised colour-blind participants). Such a categorisation

312 ensured that participants grouped in the non-colour-blind group were indeed not colour-blind

313 (had low colour blindness index scores) while participants grouped in the colour-blind group

314 were indeed relatively strongly colour-blind (i.e., had high colour blindness index scores) ${ }^{1}$.

315 There were 25 colour-blind and 25 non-colour-blind participants in the terms condition. There

316 were 24 colour-blind and 31 non-colour-blind participants in the patches condition (see Table S

317 4).

\subsection{Data Analyses}

319 We ran the subsequent analyses using these new and improved colour blindness categories. We

320 set alpha levels for all tests at .050. All analyses were two-tailed. Across statistical tests, where

321 appropriate, we controlled for familywise errors (Type I error) using False Discovery Rate

322 (FDR) correction and marked the corrected $p$-values as $p_{F D R}$ (Benjamini \& Hochberg, 1995). We

323 performed analyses and created graphs with the R v.3.4.0 and SPSS v.25.

\subsubsection{Group-Level Analyses}

325 For these analyses, we compared the re-categorised non-colour-blind and colour-blind

326 participants, as described in the section Colour Blindness Index. We continue labelling them

327 colour-blind and non-colour-blind participants, for simplicity.

\footnotetext{
${ }^{1}$ We chose the most inclusive limits. This allowed us to keep as many "real" non-colour-blind and "real" colourblind participants as possible. However, less inclusive boundaries (i.e., excluding everyone who scored between -0.7 and 0.4 on the colour blindness index) did not change the overall results of our analyses and the respective conclusions. Please find the complete dataset at https://forsbase.unil.ch/project/study-public-overview/16969/0/
} 
328 2.8.1.1. Specific Colour-Emotion Associations. We started the analyses by investigating the

329 specific emotion concepts associated with colours. We calculated the proportion of participants

330 who associated a specific emotion concept with a specific colour by dividing the number of

331 participants who chose each emotion concept for each colour by the total number of participants

332 in that group (e.g., colour-blind, terms condition). The proportion of participants was calculated

333 separately for colour-blind participants and non-colour-blind participants for each condition

334 (terms or patches) separately. The proportion values were the dependent variable, which varied

335 from 0 (very unlikely association, no one chose it) to 1 (very likely association, everyone chose 336 it).

337 To compare the pattern of emotion associations, we created four $12 \times 20$ (colours x emotions)

338 representation matrices using the proportion values to compare colour blindness groups and

339 colour presentation modes. Matrix $\mathrm{CB}_{\mathrm{CB} \text {-term }}$ contained colour-emotion associations of colour-blind

340 participants associating colour terms with emotion concepts, while Matrix $\mathrm{Non}_{\mathrm{N}-\mathrm{CB}-\mathrm{term}}$ contained

341 analogous associations of non-colour-blind participants (terms condition). Matrix $\mathrm{CB}_{\mathrm{Catch}}$

342 contained colour-emotion associations of colour-blind participants associating colour patches

343 with emotion concepts while Matrix ${ }_{\text {Non-CB-patch }}$ contained analogous associations of non-colour-

344 blind participants (patches condition; see Figure 3).

345 Then, we used Pearson matrix correlations to compare Matrix ${ }_{\mathrm{CB}-\text { term }}$ Vs. Matrix $\mathrm{X}_{\text {Non-CB-term }}$ and

346 Matrix $_{\text {CB-patch }}$ Vs. Matrix $\mathrm{N}_{\text {Non-CB-patch. These matrix correlations formed the basis for the Pattern }}$

347 Similarity Index (PSI), which reflects the degree of similarity in the pattern of colour-emotion

348 associations between two matrices. A PSI score of 1 indicates perfect pattern similarity, and a

349 PSI score of 0 indicates complete pattern dissimilarity. Furthermore, to compare the similarity of 


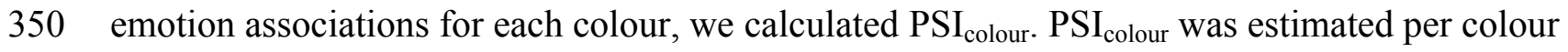

351 using Pearson correlations between colour-blind participants and non-colour-blind participants,

352 and between colour terms and colour patches.

353 To identify which colour-emotion associations differed between colour-blind and non-colour-

354 blind participants, we further used Fisher's exact tests (Fisher, 1922). The test compared the

355 proportion of participants endorsing a particular colour-emotion association (yes/no; $\mathrm{n}=240$ )

356 between colour-blind and non-colour-blind participants for terms and for patches separately. All

357 comparisons were FDR corrected (Benjamini \& Hochberg, 1995).

358 2.8.1.2. Emotion Intensity. The dependent variable emotion intensity was calculated by

359 averaging intensity ratings assigned to emotion concepts associated with each colour and for any

360 colour (i.e., “overall”). Emotion intensity varied from 1 (weak) to 5 (strong), unless no emotion

361 concept was chosen (coded as missing value).

362 A 2 x 2 independent-samples ANOVA compared average emotion intensity of all colours

363 together (i.e., "overall”) between re-categorised study groups (colour-blind vs. non-colour-blind)

364 and conditions (colour terms vs. colour patches). Afterwards, series of independent-samples $t$ -

365 tests compared emotion intensity ratings per colour between colour-blind and non-colour-blind

366 participants for terms and for patches separately, and between terms and patches for colour-blind

367 and non-colour-blind participants separately. All comparisons were FDR corrected (Benjamini \&

368 Hochberg, 1995). 
369 2.8.1.3. Supplemental Analyses. Additionally, we analysed colour associations with emotion

370 dimensions and colour naming. As these were supplementary analyses, the method and results

371 are presented in the Supplementary material.

\section{2.8.2. Individual-Level Analyses}

373 We tested whether the presence of colour-emotion associations depended on the degree of colour

374 blindness (i.e., colour blindness index). To this end, we fitted a logistic model with repeated

375 measures using glmer function in lme4 package (Bates et al., 2015). We used the likelihood ratio

376 test to test for significance of individual predictors and their interactions. The predictor variables

377 were the colour blindness index, colour presentation mode (2 levels, independent), colour (12

378 levels, repeated), and emotion (20 levels, repeated). We also tested for the three interactions

379 between the colour blindness index and i) colour presentation mode, ii) colour, and iii) emotion.

380 The outcome variable was presence of colour-emotion association (yes/no). These analyses were 381 performed on all participants $(n=129)$.

\section{3. Results}

\subsection{Colour Blindness Scoring}

384 The $2 \times 2$ MANOVA on the number of errors in colour blindness test scores indicated that

385 colour-blind participants made significantly more errors than non-colour-blind participants on all

386 tests. However, their performance did not differ by condition (terms or patches). More details are

387 presented in the Supplementary material and Table S 5.

\subsection{Group-Level Analyses}

\subsubsection{Specific Colour-Emotion Associations}


390 Some colour-emotion associations were prominent in both study groups. For instance, red-love,

391 red-anger, yellow-joy, pink-love and brown-disgust were chosen by 50\% or more of colour-blind

392 as well as non-colour-blind participants (terms and patches combined). The majority of colour-

393 blind participants also associated orange with joy, yellow with pleasure, and blue with pleasure.

394 The majority of non-colour-blind participants also associated red with pleasure, red with hate,

395 yellow with amusement, turquoise with joy and pleasure, blue with interest, pink with joy and

396 pleasure, grey with sadness and disappointment, and black with fear. See Figure 3 for visual

397 representation of all colour-emotion associations (and supplemental tables for the numeric

398 values: Table S 10, Table S 11, Table S 12, and Table S 13).

3.2.1.1. Colour-Blind vs. Non-Colour-Blind Participants. After having described the specific

400 colour-emotion associations, we compared the pattern of colour-emotion associations between

401 study groups and conditions. The matrix correlations, PSI, were overall high. PSI comparing

402 emotion associations with colour terms (Matrix CB-term $_{\text {Vs. Matrix }}$ Non-CB-term ) showed high

403 similarity, $r=.82, R^{2}=.672, p<.001$, and so did PSI comparing emotion associations with

404 patches $\left(\right.$ Matrix $_{\mathrm{CB} \text {-patch }}$ vs. Matrix $\mathrm{Non-CB-patch}_{\text {) }}, r=.80, R^{2}=.637, p<.001$ (see Figure 3). These

405 correlation coefficients were of similar strength, $z=-0.63, p=.529$. These results imply that

406 colour-blind participants and non-colour-blind participants associated similar emotions,

407 irrespective of whether a colour was presented as a term or a patch.

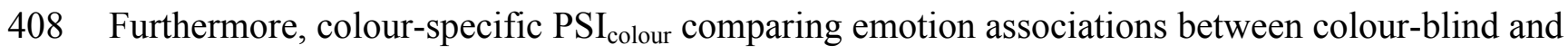
409 non-colour-blind participants for each colour were high for colour terms, $r=.60-.97, R^{2}=.355$ -

$410.939, p_{F D R}<.006$, and for colour patches, $r=.55-.92, R^{2}=.548-.924, p_{F D R}<.012$, see Table 3.

411 The only exception was purple, for colour terms, $r=.07, R^{2}=.004, p_{F D R}=.781$, and for colour 
412 patches, $r=.09, R^{2}=.007, p_{F D R}=.721$. These results indicate that the similarity between colour-

413 blind and non-colour-blind participants held across all colours, whether a term or a patch was

414 presented, with purple being an exception.

415 Fisher's exact tests were used to identify any differences between the specific colour-emotion 416 associations between the two study groups, separately for each condition. No specific colour-

417 emotion comparisons were significant suggesting that no colour-emotion specific association 418 differed between the two study groups $\left(p_{F D R} \geq .39\right)$. Thus, despite low correlations for purple,

419 we could not detect specific emotion associations driving this dissimilarity.

3.2.1.2. Colour Terms vs. Colour Patches. Furthermore, we compared the pattern of emotion associations with colour terms and colour patches, respectively, for each study group separately. The matrix-to-matrix correlations, PSI, were again overall high. PSI comparing emotion associations between colour terms and colour patches in colour-blind participants (Matrix $\mathrm{CB}_{\mathrm{CB}-\mathrm{term}}$ vs. Matrix $\mathrm{CB}_{\mathrm{CB} \text {-patch }}$ ) showed high similarity, $r=.74, R^{2}=.552, p<.001$, and so did PSI comparing emotion associations between colour terms and colour patches in non-colour-blind participants (Matrix $_{\text {Non-CB-term }}$ Vs. Matrix Non-CB-patch $\left._{\text {) }}\right), r=.83, R^{2}=.683, p<.001$ (see Figure 3). However, the correlation coefficient in colour-blind participants was significantly lower than in non-colourblind participants, $z=-2.59, p=.010$. These results mean that similar emotions were associated with colour terms and with colour patches by non-colour-blind participants as well as by colourblind participants, but the latter did so to a lower extent.

431 Furthermore, colour-specific PSI $_{\text {colour }}$ comparing emotion associations between colour terms and 432 colour patches for each colour were high for colour-blind participants, $r=.46-.89, R^{2}=.214$ -

$433.795, p_{F D R}<.040$, and for non-colour-blind participants, $r=.49-.96, R^{2}=.243-.929, p_{F D R}<.027$, 
434 see Table 3. The exception again was purple, associations of which did not correlate for colour-

435 blind participants, $r=.26, R^{2}=.066, p_{F D R}=.273$. Correlations for green in colour-blind

436 participants were significant but low $(p=.040)$. These results indicated that the similarity

437 between colour terms and colour patches was equally true for colour-blind and non-colour-blind 438 participants, with the exception of purple.

439 Fisher's exact tests were used to identify differences for specific colour-emotion associations

440 between conditions, separately for colour-blind and non-colour-blind participants. No specific

441 colour-emotion comparisons were significant $\left(p_{F D R} \geq .57\right)$. Thus, despite a low correlation in 442 colour-blind participants between purple as a patch and as a term, we could not detect specific 443 emotion associations driving this dissimilarity.

[Insert Figure 3 around here]

\subsubsection{Emotion Intensity}

449 The $2 \times 2$ ANOVA revealed a significant main effect of condition, $F(1,101)=14.8, p<.001, \eta_{\mathrm{p}}{ }^{2}$

$450=.123$, indicating that more intense emotions were associated with colour terms than colour

451 patches by both study groups. There was no significant main effect of study group, $F(1,101)=$

$4522.44, p=.121, \eta_{\mathrm{p}}{ }^{2}=.024$, indicating that colour blind and non-colour-blind participants

453 associated equally intense emotions overall. Finally, the interaction between study group and 
454 condition was not significant, $F(1,101)=0.23, p=.440, \eta_{\mathrm{p}}{ }^{2}=.006$. For differences by colour,

455 see supplemental material (Table S 6, Table S 7, Table S 8, and Table S 9).

\subsection{Individual-Level Analyses}

457 The multilevel logistic regression model was overall significant, $L R(63)=876, p<.001$, ${ }_{p s e u d o} R^{2}$ $458=.028\left(\right.$ Cox \& Snell), .047 (Nagelkerke). Both, colour, $L R(12)=161, p<.001,{ }_{p s e u d o} R^{2}=.005$

459 (Cox \& Snell), .009 (Nagelkerke), and emotion, $L R(20)=675, p<.001$, pseudo $R^{2}=.022($ Cox \&

460 Snell), .037 (Nagelkerke), were significant predictors of whether colours and emotions were

461 associated or not. In contrast, the colour blindness index was not a significant predictor of the

462 probability of colour-emotion associations, $L R(1)=0.03, p=.865,{ }_{\text {pseudo }} R^{2}<.001$ (Cox \& Snell),

$463<.001$ (Nagelkerke). Hence, the probability of colour-emotion associations did not vary by

464 degree of colour blindness. Condition was not a significant predictor either, $\operatorname{LR}(1)=0.14, p=$

$465.711,{ }_{\text {pseudo }} R^{2}<.001$ (Cox \& Snell), $<.001$ (Nagelkerke).

466 The two-way interaction between the colour blindness index and colour was significant, $L R(11)$

$467=23.4, p=.016,{ }_{\text {pseudo }} R^{2}=.001$ (Cox \& Snell), .001 (Nagelkerke). Higher colour blindness index 468 resulted in lower probability of emotion associations with $\mathrm{red}, \beta=-0.17, z=-2.08, p=.037$.

469 However, this effect was weak and disappeared after FDR correction $\left(p_{F D R}=.44\right)$. The colour

470 blindness index was not a significant predictor for other colours, $p s_{F D R}=.96$. The other two-way

471 interactions between the colour blindness index and emotion, $\operatorname{LR}(19)=9.58, p=.96$, ${ }_{\text {seudo }} R^{2}<$

$472.001($ Cox \& Snell), $<.001$ (Nagelkerke), and the colour blindness index and condition, $L R(1)=$

$4731.73, p=.189,{ }_{\text {pseudo }} R^{2}<.001$ (Cox \& Snell), $<.001$ (Nagelkerke), were not significant. 
474 Given these zero results, we wished to estimate the likelihood that, indeed, the colour blindness

475 index is unlikely to predict the probability of colour-emotion associations. We examined the key

476 predictor of interest (colour blindness index) by estimating the Bayes factor using Bayesian

477 Information Criteria (Wagenmakers, 2007; Jarosz \& Wiley, 2014). The Bayes factor compared

478 the fit of the data under the null hypothesis with the fit of the data under the alternative

479 hypothesis. The estimated Bayes factor (null/alternative; $B F_{01}$ ) was 245:1, suggesting that the

480 data were 245 times more likely to occur under the null hypothesis than the alternative

481 hypothesis. Reversely, the data were 0.004 times more likely to occur under the alternative than

482 the null hypothesis $\left(B F_{10}\right)$.

\section{4. Discussion}

484 Colours are associated with emotions (Wexner, 1954; Adams \& Osgood, 1973; Valdez \& 485 Mehrabian, 1994; Kaya \& Epps, 2004; Fugate \& Franco, 2019; Tham et al., 2019; Schloss, 486 Witzel \& Lai, 2020) and these associations might be universal across cultures (Adams \& 487 Osgood, 1973; D’Andrade \& Egan, 1974; Gao et al., 2007; Ou et al., 2018; Jonauskaite et al., 488 2020a). If the assumption on universality holds true, we have to ask whether these associations 489 originate from our shared i) conceptual, abstract understanding of the world (Xu, Dowman \& 490 Griffiths, 2013), or ii) perceptual experience of inhabiting the globe (Palmer \& Schloss, 2010).

491 Recently, Jonauskaite, et al. (2020b) showed that colour-emotion associations were similar for 492 colour patches and colour terms in young Swiss adults. These results indicate that i) conceptual 493 colour experiences seem sufficient for colour-emotion associations to occur, and ii) immediate 494 perceptual colour experiences do not seem necessary. 
495 To further assess these suggestions, we tested men with congenital red-green colour blindness as

496 well as men with intact colour vision. We tested men, because they have a much higher

497 incidence of colour blindness than women (Sharpe et al., 1999; Birch, 2012). Our participants

498 associated 12 colours with 20 emotion terms, and rated the emotion intensities (see also

499 Jonauskaite et al., 2020b). Half of our participants associated colour terms, and the other half

500 associated colour patches. Participants who associated colour patches also named them. We

501 found that colour-blind and non-colour-blind men showed a high degree of similarity in colour-

502 emotion associations, whether associating colour terms or colour patches. In case of colour

503 patches, the two groups named colours almost identically. Furthermore, the strength of colour

504 blindness neither predicted colour-emotion associations nor emotion intensities. Within group

505 comparisons showed highly similar emotion associations with terms and patches (see also

506 Jonauskaite et al., 2020b), with yet a higher similarity found in non-colour-blind than colour-

507 blind men.

508 Before discussing these major findings, we highlight that we tested representative samples. We

509 replicated common colour-emotion associations such as red-love, red-anger, yellow-joy, pink-

510 love, and brown-disgust associations (Kaya \& Epps, 2004; Fugate \& Franco, 2019; Jonauskaite

511 et al., 2019a, 2020a). When we clustered the 20 emotion concepts into the affective dimensions

512 of valence, arousal, and power, we replicated that black, grey, and brown were negative colours;

513 yellow, orange, blue, turquoise, pink, and white were positive colours; and red was an arousing

514 and powerful colour associated with both positive and negative emotions (Adams \& Osgood,

515 1973; Valdez \& Mehrabian, 1994; Soriano \& Valenzuela, 2009; Lakens, Semin \& Foroni, 2012;

516 Sutton \& Altarriba, 2016; Specker et al., 2018; Jonauskaite et al., 2020b). These colour-emotion

517 associations were endorsed by both colour-blind and non-colour-blind men. 
518 When returning to our major findings, we have to first remember that colour-blind individuals

519 perceive colours differently from non-colour-blind individuals since birth (Linhares, Pinto \&

520 Nascimento, 2008). They have diminished or completely absent excitations of the L or M

521 photoreceptors (Dalton, 1798; Parry, 2015). Second, we have to remember that colour-blind

522 individuals have learned the same conceptual representations of colour as non-colour-blind

523 individuals (Byrne \& Hilbert, 2010), including colour naming (Bonnardel, 2006, and the current

524 study). With these pieces of information in mind, we can start considering what it might mean

525 that our colour-blind and non-colour-blind participants provided highly similar colour-emotion

526 associations, despite partially different perceptual experiences. First of all, participants likely

527 activated similar abstract colour representations when reading a colour term (e.g., red) to when

528 looking at the actual colour patch. Then, we can also consider that the colour-emotion

529 associations were more majorly driven by the conceptual representations of colours, because

530 seeing actual colour patches seemed to carry no additional information to colour-emotion

531 associations (see also, Jonauskaite et al., 2020b). The latter consideration echoes analogue

532 notions for colour-tone associations (Saysani, 2019), transmission of colour terms (Xu, Dowman

533 \& Griffiths, 2013), mental colour spaces (Shepard \& Cooper, 1992; Saysani, Corballis \&

534 Corballis, 2018a,b), or object-colour knowledge (Wang et al., 2020). So far, we have to limit our

535 reasoning to colour-emotion associations for focal colours, which we presented here, and which

536 are highly recognisable by colour-blind men (see also, Moreira et al., 2014).

537 So far, we have discussed the high similarities between groups and conditions. However, the 538 degree of similarities fell short of $100 \%$, leaving space for additional variance to be explained.

539 Part of this variance might be random noise, but part might be linked to meaningful individual

540 differences. In this regard, the degree of colour blindness was uninformative; it did not explain 
541 colour-emotion associations or emotion intensities. We observed, however, that the similarity of

542 emotion associations with terms and patches was less pronounced for colour-blind than non-

543 colour-blind men. This relatively lower similarity points to a possible influence of actual colour

544 experiences to colour-emotion associations (see also, Saysani et al., 2018b; Shepard \& Cooper,

545 1992). One could suggest that colour-blind men as compared to non-colour-blind men were less

546 certain when naming colour patches. This suggestion seems unlikely, however, because colour-

547 blind and non-colour-blind men named the patches of focal colours almost identically.

548 Alternatively, due to perceptual deficiencies, colour-blind men who saw colour patches might

549 have activated slightly different abstract colour representations than colour-blind men who read

550 colour terms, especially for colours affected by colour blindness. We found that colour-blind

551 men showed the lowest patch-term similarities for purple and green, and associated more intense

552 emotion concepts with red, orange, yellow, pink, black, and white when colours were presented

553 as terms than patches (see also Jonauskaite et al., 2020b for stronger emotion intensities with

554 terms than patches). Also, colour-blind men associated fewer emotion concepts with red than

555 non-colour-blind men. Colour-blind men might have imagined these colours more vividly than

556 seen in patches, associating more intense and specific emotions when processing these terms.

557 Overall, our observations on high degrees of similarities support the previous literature, showing

558 high similarities in colour-emotion associations across cultures (Adams \& Osgood, 1973;

559 D’Andrade \& Egan, 1974; Gao et al., 2007; Ou et al., 2018; Jonauskaite et al., 2019c, 2020a). At

560 the same time, studies have also shown systematic variations on long-term and short-term scales.

561 On long-term scales, high similarities in colour-emotion associations were more pronounced

562 when individuals came from nations that were linguistically and/or geographically closer

563 (Jonauskaite et al., 2020a). For instance, individuals living closer to the equator had a lower 
564 likelihood to associate yellow with joy than individuals living further away from the equator

565 (Jonauskaite et al., 2019a). Studies have also shown systematic variations on shorter time scales.

566 Individuals living in the same nation preferred autumn-like colours more strongly in autumn than

567 during other seasons of the year (Schloss \& Heck, 2017; Schloss et al., 2017). On even shorter

568 time scales, colour preferences have been influenced in a laboratory experiment (Strauss, Schloss

$569 \&$ Palmer, 2013). These authors showed that exposure to numerous positive objects (e.g.,

570 strawberries and wine) increased the liking of the respective colour (e.g., red), while exposure to

571 numerous negative objects (e.g., a bloody nose and rotten tomatoes) decreased the liking of the

572 respective colour (e.g., red). Likely, studies showing such systematic variations demonstrate the

573 human species' abilities to adapt to particularities of their respective environments (Lupyan \&

574 Dale, 2016).

575 As an auxiliary finding, we observed a low similarity in emotion associations with purple. We

576 observed dissimilar associations between colour-blind and non-colour-blind men as well as

577 between terms and patches in colour-blind men. Colour-blind men associated purple with diverse

578 positive as well as negative emotions, while non-colour-blind men associated purple, especially

579 as a term, with positive emotions, mainly with love. Diverse findings for purple are not new.

580 Participants in general disagree which emotions purple represent, whether data originate from

581 the same nation (Wexner, 1954; Hemphill, 1996; Sandford, 2014; Sutton \& Altarriba, 2016;

582 Fugate \& Franco, 2019), from four or 30 nations (Hupka et al., 1997; Jonauskaite et al., 2020a),

583 or when comparing terms and patches (Jonauskaite et al., 2020b), as was also done here. We

584 suggest that this lack of clarity for purple is an interesting observation, so much so that it

585 deserves its own investigation (e.g., Hamilton, 2014; Oja \& Uusküla, 2011; Tager, 2018). 


\subsection{Strengths and Limitations}

587 There are numerous strengths and limitations to our study. The first strength is that we employed

588 the same method used previously to assess colour-emotion associations (Griber, Jonauskaite \&

589 Mohr, 2019; Jonauskaite et al., 2019a,b,c, 2020a,b). This consistency simplifies direct

590 comparisons between studies. The second strength is that we recruited a large number of

591 congenitally colour-blind men, at least when comparing our sample size to previous studies

592 (Shepard \& Cooper, 1992; Paramei, 1996; Paramei, Bimler \& Cavonius, 1998; Bonnardel, 2006;

593 Moreira et al., 2014; Álvaro et al., 2015, 2017; Sato \& Inoue, 2016; Saysani, Corballis \&

594 Corballis, 2018a). By default, a larger sample size provides more representative colour-emotion

595 associations. Yet, having a larger sample size for our colour-blind men also meant that our

596 sample was relatively diverse (see also Bonnardel, 2006; Nagy et al., 2014; Paramei, 1996). We

597 recruited all men who had self-reported congenital red-green colour blindness, irrespective of its

598 strength. Thus, we tested men with partial as well as complete colour vision deficiencies (i.e.,

599 dichromatic and anomalous trichromatic vision), with mainly deutan-like or unidentified

600 impairments. Only some previous studies aimed for a sample of exclusively dichromatic

601 participants (e.g., Álvaro et al., 2015, 2017; Moreira et al., 2014; Shepard \& Cooper, 1992),

602 resulting in a much smaller number of tested individuals.

603 To factor in this diversity and to account for varying strength of colour blindness, we derived the

604 Colour Blindness Index from scores on three behavioural colour vision tests (Farnsworth, 1947;

605 Lanthony, 1978b; Ishihara, 2000). This Colour Blindness Index was not a significant predictor of

606 colour-emotion associations, while between- as well as within-group similarities were high.

607 Therefore, we argue that differences in colour perception within our colour-blind group bore 
608 little relevance to colour-emotion associations, at least when working with highly recognisable

609 focal colours. If this conclusion holds true, similar colour-emotion associations should also arise

610 in congenitally blind individuals. Previous studies have demonstrated that congenitally blind

611 individuals possess similar mental spaces of colour (Saysani, Corballis \& Corballis, 2018b),

612 associate similar colours with pure tones (Saysani, 2019), and represent object-colour knowledge

613 in similar brain regions as sighted individuals (Wang et al., 2020). Some blind individuals also

614 associate similar colours with semantic scales, but there is high a variability among the blind

615 (Saysani, Corballis \& Corballis, 2021).

616 Another potential limitation is the use of focal colours (i.e., best examples of colour categories)

617 and basic colour terms, both of which are overlearned. Testing colour patches that are difficult to

618 name or using non-basic colour terms, like lavender or mauve, would be the next step in this type

619 of research. Such colour stimuli might be more powerful to reveal more differences between

620 colour-blind and non-colour-blind individuals. The perceptual experience might be more

621 important when working with stimuli that are less overlearned. In a previous study (Saysani,

622 Corballis \& Corballis, 2018a), the mental arrangement of non-basic colour terms was less similar

623 between colour-blind and non-colour-blind individuals than the mental arrangement of the basic

624 colour terms. Yet, the similarity between the two groups was still very high in both conditions,

625 suggesting that colour-blind participants have a common understanding of non-basic colour 626 terms too.

\subsection{Theoretical and Practical Implications}

628 All results considered, we conclude that cultural knowledge, transmitted through language, plays 629 a sufficient role for colour-emotion associations to occur, while immediate perceptual colour 
630 experience in adulthood does not seem to be necessary. This conclusion has implications to

631 theories in which the importance of colour perception to affective associations with colour is

632 highlighted (Hurlbert \& Ling, 2007; Palmer \& Schloss, 2010; Schloss, 2018). According to the

633 cone-opponent theory (Hurlbert \& Ling, 2007), human colour preferences are influenced by

634 weights on the two cone-opponent contrast components (i.e., L-M; S- $(\mathrm{L}+\mathrm{M})$ ). According to the

635 Ecological Valence Theory (Palmer \& Schloss, 2010), human colour preferences are driven by

636 the valence of objects of the same colour. For instance, people like colours that are associated

637 with positive objects and dislike colours that are associated with negative objects. As an

638 example, blue would be liked because it is associated with clear sky and clean water while brown

639 would be disliked because it is associated with rotten food. Note, these theories have been

640 developed to explain colour preferences and not colour-emotion associations (but see Schloss,

641 2018). Perhaps, colour preferences and colour-emotion associations are guided by different

642 mechanisms. In fact, colour preferences have been hypothesised (Schloss, 2015) and empirically

643 demonstrated (Álvaro et al., 2015; Baek et al., 2015; Sato \& Inoue, 2016) to differ between

644 colour-blind and non-colour-blind individuals. More specifically, colour-blind individuals

645 preferred yellowish colours to a greater extent and bluish colours to a lesser extent than non-

646 colour-blind individuals (Álvaro et al., 2015). Colour preferences seem also less universal

647 (Taylor, Clifford \& Franklin, 2013; Schloss \& Palmer, 2017; Groyecka et al., 2019). Thus,

648 immediate perceptual experiences might be more relevant to colour preferences than to colour-

649 emotion associations. Alternatively, future theories should account for more conceptual,

650 knowledge- and language-based factors when explaining colour preferences (see Yokosawa et

651 al., 2016 for the importance of symbolic colour associations to colour preferences). 
652 If immediate perceptual experiences are not necessary for colour-emotion associations in

653 adulthood, then research on colour-emotion associations might not easily translate to applied

654 domains. For instance, proponents of colour therapy, or chromotherapy, assume that perception

655 of colour can impact one's affective states (Azeemi \& Raza, 2005; O'Connor, 2011; Winkler,

656 2012; Gul, Nadeem \& Aslam, 2015). Often, such claims are based on conceptual colour

657 associations. One can read, "Being the lightest hue of the spectrum, the colour psychology of

658 yellow is uplifting and illuminating, offering hope, happiness, cheerfulness and fun" (Scott-

659 Kemmis, 2018). Yellow was indeed conceptually associated with joy in 55 countries (Jonauskaite

660 et al., 2019a). However, an association between yellow and joy does not immediately imply that

661 looking at yellow walls or yellow objects would make one feel joyful. Empirical studies have

662 struggled to confirm many of the expected psychological effects of colour, such as pink reducing

663 aggressiveness in prisoners (Genschow et al., 2015), or pink, red, or blue enhancing cognitive

664 performance and improving mood (von Castell et al., 2018). A recent study also demonstrated

665 that direct exposure to colour was not important to stress and anxiety reduction following a

666 colour intervention (Jonauskaite et al., 2020c). In short, conceptual colour-emotion associations

667 should not be equated with and might not translate to psychological consequences of colour.

\section{5. Conclusions}

669 We evaluated whether conceptual mechanisms are sufficient for consistent colour-emotion

670 associations to emerge or whether immediate colour experience is necessary We found that

671 colour-emotion associations were highly similar between individuals with congenital red-green

672 colour blindness and individuals with intact colour vision. This high similarity was observed

673 whether colours were shown as terms or patches. Based on our findings, we conjecture that intact 
674 immediate colour vision is not necessary for colour-emotion associations, at least not in

675 adulthood. Likely, these associations are driven by conceptual mechanisms, our language and

676 knowledge. In other words, it is unlikely that colour-emotion associations arise exclusively from

677 direct affective experiences when seeing colours, because conceptual knowledge is already well

678 established. To reason one step further, high similarities between colour-blind and non-colour-

679 blind individuals as well as similarities across cultures (Adams \& Osgood, 1973; Jonauskaite et 680 al., 2020a) would suggest that colour-emotion associations present another human psychological 681 universal (Norenzayan \& Heine, 2005).

\section{6. Acknowledgements}

683 We wish to thank Amer Chamseddine for programming the first experiment and Guillaume 684 Sierro for programming the second experiment.

685 7. References

686 Adams FM, Osgood CE. 1973. A cross-cultural study of the affective meanings of color. Journal 687 of Cross-Cultural Psychology 4:135-157. DOI: 10.1177/002202217300400201.

688 Almustanyir A, Hovis JK. 2020. Color vision defectives' experience: When white is green.

689 Color Research \& Application 45:586-590. DOI: 10.1002/col.22499.

690 Álvaro L, Linhares JMM, Moreira H, Lillo J, Nascimento SMC. 2017. Robust colour constancy

691 in red-green dichromats. PLoS ONE 12:1-17. DOI: 10.1371/journal.pone.0180310.

692 Álvaro L, Moreira H, Lillo J, Franklin A. 2015. Color preference in red-green dichromats.

693 Proceedings of the National Academy of Sciences 112:9316-9321. DOI:

$694 \quad 10.1073 /$ pnas. 1502104112. 
695 Azeemi STY, Raza SM. 2005. A critical analysis of chromotherapy and its scientific evolution.

696 Evidence-based Complementary and Alternative Medicine 2:481-488. DOI:

$697 \quad 10.1093 /$ ecam/neh137.

698 Baek Y, Kwak Y, Woo S, Park C. 2015. Preferred memory color difference between the

699 deuteranomalous and normal color vision. In: Eschbach R, Marcu GG, Rizzi A eds. Color

700 Imaging XX: Displaying, Processing, Hardcopy, and Applications. 939517. DOI:

$701 \quad 10.1117 / 12.2081958$.

702 Bates D, Mächler M, Bolker B, Walker S. 2015. Fitting linear mixed-effects models using lme4. Journal of Statistical Software 67:201-210. DOI: 10.18637/jss.v067.i01.

Benjamini Y, Hochberg Y. 1995. Controlling the false discovery rate: A practical and powerful 705 approach to multiple testing. Journal of the Royal Statistical Society. Series B

706 (Methodological) 57:289-300. DOI: 10.2307/2346101.

Biggam CP. 2012. Basic colour categories. In: The semantics of colour: A historical approach. Cambridge: Cambridge University Press, 58-69.

Birch J. 2012. Worldwide prevalence of red-green color deficiency. Journal of the Optical Society of America A 29:313. DOI: 10.1364/JOSAA.29.000313.

711 Bonnardel V. 2006. Color naming and categorization in inherited color vision deficiencies. Visual Neuroscience 23:637-643. DOI: 10.1017/S0952523806233558.

713 Brainard DH, Pelli DG, Robson T. 2002. Display characterization. In: Hornak J ed. 714 Encyclopedia of imaging science and technology. Hoboken, NJ: John Wiley \& Sons, Inc., 715 172-188. DOI: 10.1002/0471443395.img011.

716 Byrne A, Hilbert DR. 2010. How do things look to the color-blind. Color Ontology and Color 
718

720

721

722

723

724

725

726

727

728

729

730

731

732

733

734

735

736

737

738

von Castell C, Stelzmann D, Oberfeld D, Welsch R, Hecht H. 2018. Cognitive performance and emotion are indifferent to ambient color. Color Research \& Application 43:65-74. DOI: 10.1002/col.22168.

D’Andrade R, Egan M. 1974. The colors of emotion. American Ethnologist 1:49-63. DOI: 10.1525/ae.1974.1.1.02a00030.

Dalton J. 1798. Extraordinary facts relating to the vision of colours: with observations. Memoirs of the Manchester Literary and Philosophical Society 5:28-45.

Farnsworth D. 1947. The Farnsworth dichotomous test for color blindness - panel D15. New York, NY: The Psychological Corp.

Faul F, Erdfelder E, Lang A-G, Buchner A. 2007. G*Power: A flexible statistical power analysis program for the social, behavioral, and biomedical sciences. Behavior Research Methods 39:175-191. DOI: 10.3758/BF03193146.

Fisher RA. 1922. On the interpretation of $\chi 2$ from contingency tables, and the calculation of $P$. Journal of the Royal Statistical Society 85:87-94. DOI: 10.2307/2340521.

Fontaine JRJ. 2013. Dimensional, basic emotion, and componential approaches to meaning in psychological emotion research. In: Components of emotional meaning: A sourcebook. Oxford: Oxford University Press, 31-45. DOI: 10.1093/acprof:oso/9780199592746.003.0003.

Forbes I. 1979. The terms brun and marron in modern standard French. Journal of Linguistics 15:295-305. DOI: 10.1017/S0022226700016406.

Fugate JMB, Franco CL. 2019. What color is your anger? Assessing color-emotion pairings in

Peer] reviewing PDF | (2020:11:55612:2:0:NEW 3 Mar 2021) 
English speakers. Frontiers in Psychology 10:1-17. DOI: 10.3389/fpsyg.2019.00206.

740 Gao X-P, Xin JH, Sato T, Hansuebsai A, Scalzo M, Kajiwara K, Guan S-S, Valldeperas J, Lis

741 MJ, Billger M. 2007. Analysis of cross-cultural color emotion. Color Research \&

742 Application 32:223-229. DOI: 10.1002/col.20321.

743 Genschow O, Noll T, Wänke M, Gersbach R. 2015. Does Baker-Miller pink reduce aggression

744 in prison detention cells? A critical empirical examination. Psychology, Crime \& Law

745 21:482-489. DOI: 10.1080/1068316X.2014.989172.

746 Griber YA, Jonauskaite D, Mohr C. 2019. Cveta ehmocij : ehksperimental'noe issledovanie

747 associativnyx svyazej v sovremennom russkom yazyke [The Colors of emotions:

748 Experimental research of associative relations in modern Russian]. Litera 8:69-86.

749 Groyecka A, Witzel C, Butovskaya M, Sorokowski P. 2019. Similarities in color preferences

750 between women and men: The case of Hadza, the hunter-gatherers from Tanzania.

751 Perception 48:428-436. DOI: 10.1177/0301006619840937.

752 Gul S, Nadeem RK, Aslam A. 2015. Chromo therapy- An effective treatment option or just a

753 myth?? Critical analysis on the effectiveness of chromo therapy. American Research

754 Journal of Pharmacy 1:62-70. DOI: 10.21694/2380-5706.15002.

755 Hamilton R. 2014. Exploring the metaphorical use of colour with the Historical Thesaurus of

756 English. A case study of purple and lavender. In: Colour Studies. Amsterdam: John

757 Benjamins Publishing Company, 153-166. DOI: 10.1075/z.191.10ham.

758 Hemphill M. 1996. A note on adults' color-emotion associations. The Journal of Genetic

759 Psychology 157:275-280. DOI: 10.1080/00221325.1996.9914865.

760 Hovis JK, Ramaswamy S, Anderson M. 2004. Repeatability indices for the Farnsworth D-15 
test. Visual Neuroscience 21:449-453. DOI: 10.1017/S0952523804213402.

762 Hupka RB, Zaleski Z, Otto J, Reidl L, Tarabrina N V. 1997. The colors of anger, envy, fear, and 763 jealousy. Journal of Cross-Cultural Psychology 28:156-171. DOI:

764 10.1177/0022022197282002.

765

766

767

768

769

770

771

772

773

774

775

776

777

778

779

780

781

782

Hurlbert AC, Ling Y. 2007. Biological components of sex differences in color preference. Current Biology 17:623-625. DOI: 10.1016/j.cub.2007.06.022.

Ishihara S. 2000. The series of plates designed as a test for color deficiency. Concise edition. Tokyo: Kanehara \& Co (Original work published 1917).

Jameson D, Hurvich LM. 1978. Dichromatic color language: "Reds" and "Greens" don't look alike but their colors do. Sensory processes 2:146-55.

Jarosz AF, Wiley J. 2014. What are the odds? A practical guide to computing and reporting Bayes Factors. The Journal of Problem Solving 7:2-9. DOI: 10.7771/1932-6246.1167.

Jonauskaite D, Abdel-Khalek AM, Abu-Akel A, Al-Rasheed AS, Antonietti J-P, Ásgeirsson ÁG, Atitsogbe KA, Barma M, Barratt D, Bogushevskaya V, Bouayed Meziane MK, Chamseddine A, Charernboom T, Chkonia E, Ciobanu T, Corona V, Creed A, Dael N, Daouk H, Dimitrova N, Doorenbos CB, Fomins S, Fonseca-Pedrero E, Gaspar A, Gizdic A, Griber YA, Grimshaw GM, Hasan AA, Havelka J, Hirnstein M, Karlsson BSA, Katembu S, Kim J, Konstantinou N, Laurent E, Lindeman M, Manav B, Marquardt L, Mefoh P, Mroczko-Wąsowicz A, Mutandwa P, Ngabolo G, Oberfeld D, Papadatou-Pastou M, Perchtold CM, Pérez-Albéniz A, Pouyan N, Rashid Soron T, Roinishvili M, Romanyuk L, Salgado Montejo A, Sultanova A, Tau R, Uusküla M, Vainio S, Vargas-Soto V, Volkan E, Wąsowicz G, Zdravković S, Zhang M, Mohr C. 2019a. The sun is no fun without rain: 
783 Physical environments affect how we feel about yellow across 55 countries. Journal of $784 \quad$ Environmental Psychology 66:101350. DOI: 10.1016/j.jenvp.2019.101350.

785 Jonauskaite D, Abu-Akel A, Dael N, Oberfeld D, Abdel-Khalek AM, Al-Rasheed AS, Antonietti 786 J-P, Bogushevskaya V, Chamseddine A, Chkonia E, Corona V, Fonseca-Pedrero E, Griber 787 YA, Grimshaw G, Hasan AA, Havelka J, Hirnstein M, Karlsson BSA, Laurent E, Lindeman 788 M, Marquardt L, Mefoh P, Papadatou-Pastou M, Pérez-Albéniz A, Pouyan N, Roinishvili 789 M, Romanyuk L, Salgado Montejo A, Schrag Y, Sultanova A, Uusküla M, Vainio S, 790 Wąsowicz G, Zdravković S, Zhang M, Mohr C. 2020a. Universal Patterns in Color791 Emotion Associations Are Further Shaped by Linguistic and Geographic Proximity. 792 Psychological Science 31:1245-1260. DOI: 10.1177/0956797620948810.

793

Jonauskaite D, Dael N, Chèvre L, Althaus B, Tremea A, Charalambides L, Mohr C. 2019b. Pink for girls, red for boys, and blue for both genders: Colour preferences in children and adults. Sex Roles 80:630-642. DOI: 10.1007/s11199-018-0955-z.

Jonauskaite D, Parraga CA, Quiblier M, Mohr C. 2020b. Feeling blue or seeing red? Similar patterns of emotion associations with colour patches and colour terms. i-Perception 11:124. DOI: $10.1177 / 2041669520902484$.

Jonauskaite D, Tremea I, Bürki L, Diouf CN, Mohr C. 2020c. To see or not to see: Importance of color perception to color therapy. Color Research \& Application 45:450-464. DOI: 10.1002/col.22490.

Jonauskaite D, Wicker J, Mohr C, Dael N, Havelka J, Papadatou-Pastou M, Zhang M, Oberfeld D. 2019c. A machine learning approach to quantify the specificity of colour-emotion associations and their cultural differences. Royal Society Open Science 6:190741. DOI: 10.1098/rsos.190741. 
806 Judd DB. 1949. Color perceptions of deuteranopic and protanopic observers. Journal of the $807 \quad$ Optical Society of America 39:252. DOI: 10.1364/JOSA.39.000252.

808 Kaya N, Epps HH. 2004. Relationship between color and emotion: a study of college students. 809 College Student Journal 38:396-406.

810 Lakens D, Semin GR, Foroni F. 2012. But for the bad, there would not be good: Grounding

811 valence in brightness through shared relational structures. Journal of Experimental

$812 \quad$ Psychology: General 141:584-594. DOI: 10.1037/a0026468.

813 Lanthony P. 1978a. The new color test. Documenta Ophthalmologica 46:191-199. DOI:

$814 \quad 10.1007 / \mathrm{BF} 00174108$.

815 Lanthony P. 1978b. The desaturated panel D-15. Documenta Ophthalmologica 46:185-189.

$816 \quad$ DOI: $10.1007 / B F 00174107$.

817 Lindsey DT, Brown AM. 2014. The color lexicon of American English. Journal of Vision 14:17.

$818 \quad$ DOI: $10.1167 / 14.2 .17$.

819 Linhares JMMMM, Pinto PD, Nascimento SMCMC. 2008. The number of discernible colors

820 perceived by dichromats in natural scenes and the effects of colored lenses. Visual

$821 \quad$ Neuroscience 25:493-499. DOI: 10.1017/S0952523808080620.

822 Lupyan G, Dale R. 2016. Why are there different languages? The role of adaptation in linguistic 823 diversity. Trends in Cognitive Sciences 20:649-660. DOI: 10.1016/j.tics.2016.07.005.

824 Madden TJ, Hewett K, Roth MS. 2000. Managing images in different cultures: A cross-national 825 study of color meanings and preferences. Journal of International Marketing 8:90-107.

826 DOI: $10.1509 /$ jimk.8.4.90.19795.

827 Mohr C, Jonauskaite D, Dan-Glauser ES, Uusküla M, Dael N. 2018. Unifying research on colour 
and emotion: Time for a cross-cultural survey on emotion associations with colour terms.

829 In: MacDonald LW, Biggam CP, Paramei G V eds. Progress in colour studies: Cognition,

830 language, and beyond. Amsterdam: John Benjamins Publishing Company, 209-222. DOI:

$831 \quad 10.1075 /$ z. $217.11 \mathrm{moh}$.

832 Moreira H, Lillo J, Álvaro L, Davies I. 2014. Use of basic color terms by red-green dichromats.

833 II. Models. Color Research and Application 39:372-386. DOI: 10.1002/col.21802.

834 Nagy B V., Németh Z, Samu K, Ábrahám G. 2014. Variability and systematic differences in

835 normal, protan, and deutan color naming. Frontiers in Psychology 5:1-7. DOI:

$836 \quad 10.3389 /$ fpsyg.2014.01416.

837 Neitz M, Neitz J. 2000. Molecular genetics of color vision and color vision defects. Archives of 838 Ophthalmology 118:691-700. DOI: 10.1001/archopht.118.5.691.

839 Norenzayan A, Heine SJ. 2005. Psychological universals: What are they and how can we know?

$840 \quad$ Psychological Bulletin 131:763-784. DOI: 10.1037/0033-2909.131.5.763.

841 O’Connor Z. 2011. Colour psychology and colour therapy: Caveat emptor. Color Research and 842 Application 36:229-234. DOI: 10.1002/col.20597.

843 Oja V, Uusküla M. 2011. Indo-European influence on Finnic colour naming and categorisation.

844 In: Varella S ed. Languages and Cultures in Contact and Contrast: Historical and

845 Contemporary Perspectives. Atiner, 7-18.

846 Ou L-C, Yuan Y, Sato T, Lee W-YY, Szabó F, Sueeprasan S, Huertas R. 2018. Universal models

847 of colour emotion and colour harmony. Color Research and Application 43:736-748. DOI:

$848 \quad 10.1002 /$ col.22243.

849 Palmer SE, Schloss KB. 2010. An ecological valence theory of human color preference. 

10.1073/pnas.0906172107.

852 Paramei G V. 1996. Color space of normally sighted and color-deficient observers reconstructed 853 from color naming. Psychological Science 7:311-317. DOI: 10.1111/j.14679280.1996.tb00380.x.

855 Paramei G V., Bimler DL, Cavonius CR. 1998. Effect of luminance on color perception of 856 protanopes. Vision Research 38:3397-3401. DOI: 10.1016/S0042-6989(97)00454-9.

857 Parry NRA. 2015. Color vision deficiencies. In: Elliot AJ, Fairchild MD, Franklin A eds. 858 Handbook of Color Psychology. Cambridge: Cambridge University Press, 216-242. DOI: $859 \quad 10.1017 /$ CBO9781107337930.011.

860 Sandford JL. 2014. Turn a colour with emotion: a linguistic construction of colour in English. $861 \quad$ Journal of the International Colour Association 13:67-83.

862 Sato K, Inoue T. 2016. Perception of color emotions for single colors in red-green defective 863 observers. PeerJ 4:e2751. DOI: 10.7717/peerj.2751.

864 Saysani A. 2019. How the blind hear colour. Perception 48:237-241. DOI:

$865 \quad 10.1177 / 0301006619830940$.

866 Saysani A, Corballis MC, Corballis PM. 2018a. The colour of words: how dichromats construct 867 a colour space. Visual Cognition 26:601-607. DOI: 10.1080/13506285.2018.1524804.

868 Saysani A, Corballis MC, Corballis PM. 2018b. Colour envisioned: concepts of colour in the 869 blind and sighted. Visual Cognition 26:382-392. DOI: 10.1080/13506285.2018.1465148.

870 Saysani A, Corballis MC, Corballis PM. 2021. Seeing colour through language: Colour 871 knowledge in the blind and sighted. Visual Cognition 29:63-71. DOI: 
873 Scherer KR. 2005. What are emotions? And how can they be measured? Social Science 874 Information 44:695-729. DOI: 10.1177/0539018405058216.

875 Scherer KR, Shuman V, Fontaine JRJ, Soriano C. 2013. The GRID meets the Wheel: Assessing 876 emotional feeling via self-report. In: Fontaine JRJ, Scherer KR, Soriano C eds. Components 877 of emotional meaning: A sourcebook. Oxford: Oxford University Press, 281-298. DOI: $878 \quad$ 10.13140/RG.2.1.2694.6406.

879 Schloss KB. 2015. Color Preferences Differ with Variations in Color Perception. Trends in $880 \quad$ Cognitive Sciences 19:554-555. DOI: 10.1016/j.tics.2015.08.009.

881 Schloss KB. 2018. Chapter 6. A Color Inference Framework. Progress in Colour Studies:107882 122. DOI: $10.1075 /$ z.217.06sch.

883 Schloss KB, Heck IA. 2017. Seasonal changes in color preferences are linked to variations in 884 environmental colors: A longitudinal study of fall. i-Perception 8:1-19. DOI: $10.1177 / 2041669517742177$.

886 Schloss KB, Nelson R, Parker L, Heck IA, Palmer SE. 2017. Seasonal variations in color 887 preference. Cognitive Science 41:1589-1612. DOI: 10.1111/cogs.12429.

888 Schloss KB, Palmer SE. 2017. An ecological framework for temporal and individual differences 889 in color preferences. Vision Research 141:95-108. DOI: 10.1016/j.visres.2017.01.010.

890 Schloss KB, Witzel C, Lai LY. 2020. Blue hues don't bring the blues: questioning conventional 891 notions of color-emotion associations. Journal of the Optical Society of America A 37:813. 892 DOI: $10.1364 /$ JOSAA.383588.

893 Scholtyssek C, Kelber A, Dehnhardt G. 2014. Why do seals have cones? Behavioural evidence 
894 for colour-blindness in harbour seals. Animal Cognition 18:551-560. DOI: 10.1007/s10071$895 \quad 014-0823-3$.

896 Scott-Kemmis J. 2018.The Color Yellow. Available at https://www.empower-yourself-with897 color-psychology.com/color-yellow.html (accessed April 17, 2020).

898 Sharpe LT, Stockman A, Jägle H, Nathans J. 1999. Opsin genes, cone photopigments, color 899 vision, and color blindness. In: Gegenfurtner KR, Sharpe LT eds. Color Vision: From $900 \quad$ Genes to Perception. Cambridge: Cambridge University Press, 3-52.

901 Shepard RN, Cooper LA. 1992. Representation of colors in the blind, color-blind, and normally 902 sighted. Psychological Science 3:97-104. DOI: https://doi.org/10.1111/j.1467$903 \quad$ 9280.1992.tb00006.x.

904 Siniscalchi M, D’Ingeo S, Fornelli S, Quaranta A. 2017. Are dogs red-green colour blind? Royal 905 Society Open Science 4:170869. DOI: 10.1098/rsos.170869.

906 Soriano C, Fontaine JRJ, Scherer KR, Akırmak GA, Alarcón P, Alonso-Arbiol I, Bellelli G, 907 Pérez-Aranibar CC, Eid M, Ellsworth P, Galati D, Hareli S, Hess U, Ishii K, Jonker C, 908 Lewandowska-Tomaszczyk B, Meiring D, Mortillaro M, Niiya Y, Ogarkova A, Panasenko 909 N, Ping H, Protopapas A, Realo A, Ricci-Bitti PE, Shen Y-L, Sheu C-F, Siiroinen M, Sunar

910 D, Tissari H, Tong EMW, van Osch Y, Wong S, Yeung DY, Zitouni A. 2013. Cross-

911 cultural data collection with the GRID instrument. In: Components of emotional meaning: A 912 sourcebook. Oxford: Oxford University Press, 98-105. DOI:

913 10.1093/acprof:oso/9780199592746.003.0007.

914 Soriano C, Valenzuela J. 2009. Emotion and colour across languages: implicit associations in 915 Spanish colour terms. Social Science Information 48:421-445. DOI: 
916 $10.1177 / 0539018409106199$.

917 Specker E, Leder H, Rosenberg R, Hegelmaier LM, Brinkmann H, Mikuni J, Kawabata H. 2018.

918 The universal and automatic association between brightness and positivity. Acta

919 Psychologica 186:47-53. DOI: 10.1016/j.actpsy.2018.04.007.

920 Spence NCW. 1989. The Linguistic Field of Colour Terms in French. Zeitschrift fur Romanische 921 Philologie 105:472-497. DOI: 10.1515/zrph.1989.105.5-6.472.

922 Strauss ED, Schloss KB, Palmer SE. 2013. Color preferences change after experience with 923 liked/disliked colored objects. Psychonomic bulletin \& review 20:935-43. DOI:

924 10.3758/s13423-013-0423-2.

925

926

927

928

929

930

931

932

933

934

935

936

937
Sutton TM, Altarriba J. 2016. Color associations to emotion and emotion-laden words: A collection of norms for stimulus construction and selection. Behavior research methods 48:686-728. DOI: 10.3758/s13428-015-0598-8.

Tager A. 2018. Why was the color violet rarely used by artists before the 1860s? Journal of Cognition and Culture 18:262-273. DOI: 10.1163/15685373-12340030.

Taylor C, Clifford A, Franklin A. 2013. Color preferences are not universal. Journal of Experimental Psychology: General 142:1015-1027. DOI: 10.1037/a0030273.

Tham DSY, Sowden PT, Grandison A, Franklin A, Lee AKW, Ng M, Park J, Pang W, Zhao J. 2019. A systematic investigation of conceptual color associations. Journal of Experimental Psychology: General. DOI: 10.1037/xge0000703.

Thiadens AAHJ, Hoyng CB, Polling JR, Bernaerts-Biskop R, van den Born LI, Klaver CCW. 2013. Accuracy of four commonly used color vision tests in the identification of cone disorders. Ophthalmic Epidemiology 20:114-122. DOI: 10.3109/09286586.2012.759596.

PeerJ reviewing PDF | (2020:11:55612:2:0:NEW 3 Mar 2021) 
938 Valdez P, Mehrabian A. 1994. Effects of color on emotions. Journal of Experimental

939 Psychology: General 123:394-409. DOI: 10.1037/0096-3445.123.4.394.

940 Wagenmakers E-J. 2007. A practical solution to the pervasive problems ofp values. Psychonomic

$941 \quad$ Bulletin \& Review 14:779-804. DOI: 10.3758/BF03194105.

942 Wang X, Men W, Gao J, Caramazza A, Bi Y. 2020. Two forms of knowledge representations in 943 the human brain. Neuron:691931. DOI: 10.1016/j.neuron.2020.04.010.

944 Wang T, Shu S, Mo L. 2014. Blue or red? The effects of colour on the emotions of Chinese 945 people. Asian Journal of Social Psychology 17:152-158. DOI: 10.1111/ajsp.12050.

946 Wexner LB. 1954. The degree to which colors (hues) are associated with mood-tones. Journal of $947 \quad$ Applied Psychology 38:432-435. DOI: 10.1037/h0062181.

948 Winkler S. 2012.How does color therapy work? Available at

949 https://health.howstuffworks.com/wellness/spa-health/color-therapy.htm (accessed January $950 \quad 11,2019)$.

951 World Medical Association. 2013. World Medical Association declaration of Helsinki. Ethical 952 principles for medical research involving human subjects. The Journal of the American 953 Medical Association 310:2191-2194. DOI: 10.1001/jama.2013.281053.

954 Xu J, Dowman M, Griffiths TL. 2013. Cultural transmission results in convergence towards 955 colour term universals. Proceedings of the Royal Society B: Biological Sciences $956 \quad 280: 20123073$. DOI: $10.1098 / \mathrm{rspb} .2012 .3073$.

957 Yokosawa K, Schloss KB, Asano M, Palmer SE. 2016. Ecological effects in cross-cultural 958 differences between U.S. and Japanese color preferences. Cognitive Science 40:1-27. DOI: $959 \quad 10.1111 / \operatorname{cogs} .12291$. 
Figure 1

Geneva Emotion Wheel (GEW) to assess colour-emotion associations with colour terms and colour patches.

The intensity of the associated emotions was assessed with circles of increasing size, smaller circles indicated less intense emotions. See Table S 1. for the French version (Scherer, 2005; Scherer et al., 2013).

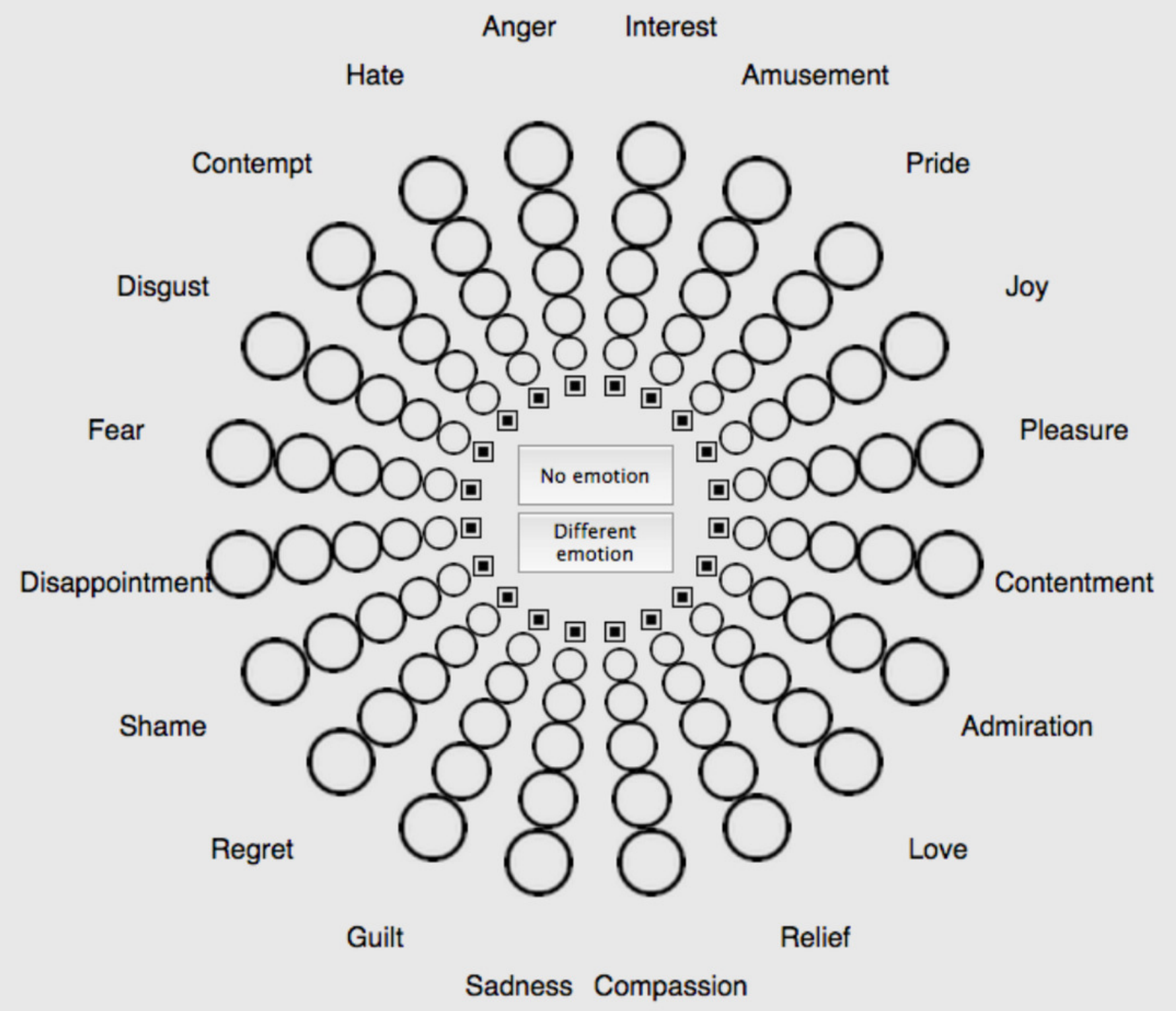




\section{Figure 2}

Procedure for the colour terms and colour patches conditions.

(A) Participants received written study information and signed informed consent. (B)

Participants completed the Ishihara test. (C) Main experiment. In Experiment 1, participants saw 12 colour terms in randomised order. They associated colour terms with one, several, or none of the Geneva Emotion Wheel (GEW) emotion concepts (see Emotion assessment and Figure 1 for enlarged GEW). In the patches condition, participants saw 12 colour patches in randomised order. They associated colour patches with one, several, or none of the GEW emotion concepts on the subsequent screen. Here, they saw the small GEW squares as well as the GEW rays of chosen emotion concepts presented in the colour they were currently evaluating. In both conditions, participants answered demographic questions. (D) In the patches condition, most participants also performed a colour-naming task. (E) Participants completed the Farnsworth D-15 and Lanthony D-15 tests in random order. (F) Participants were debriefed.

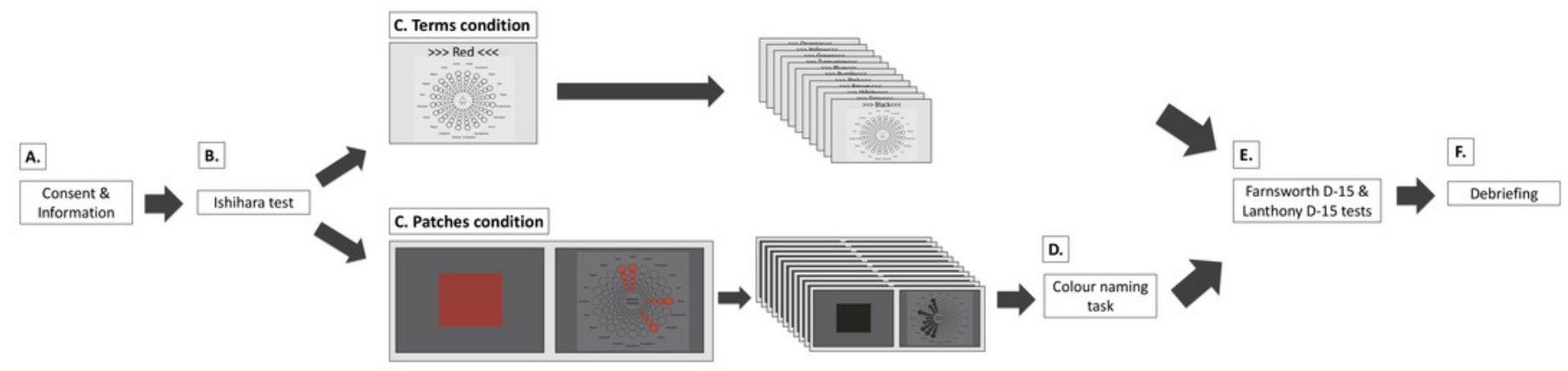




\section{Figure 3}

Heatmaps of colour-emotion associations

(A) Colour-emotion associations with colour terms in colour-blind participants; (B) Colouremotion associations with colour patches in colour-blind participants; (C) Colour-emotion associations with colour terms in non-colour-blind participants; (C) Colour-emotion associations with colour patches in non-colour-blind participants. Redder cells indicate higher proportions of participants choosing specific colour-emotion associations. 
Terms: Colour-blind

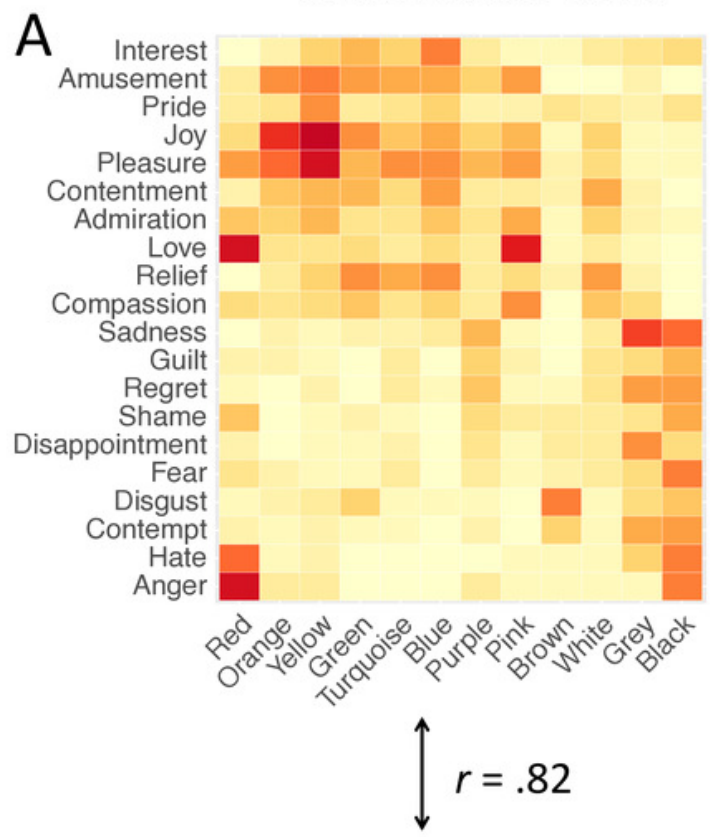

Terms: Non-colour-blind

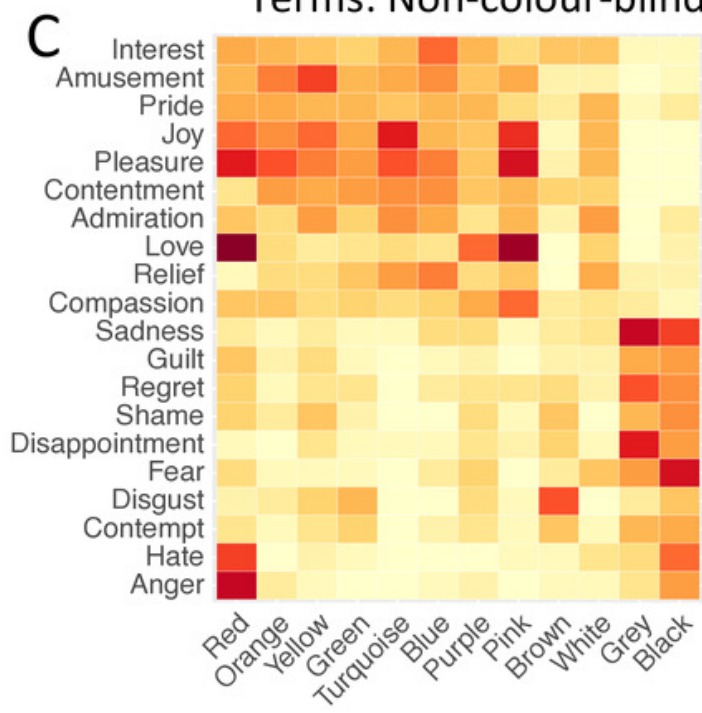

Patches: Colour-blind

B

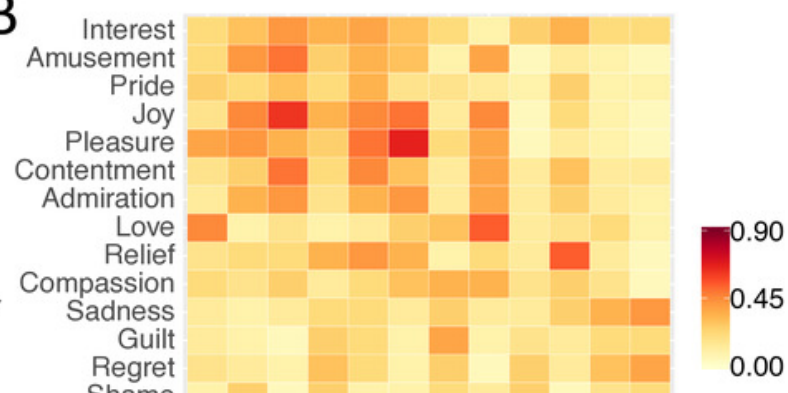

Disappointment

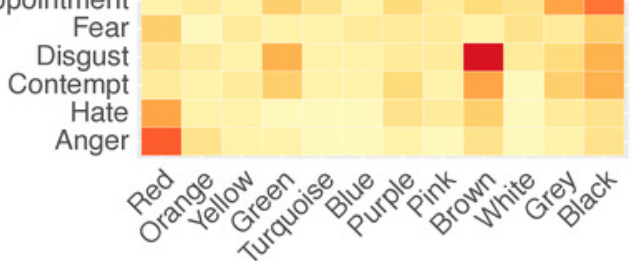

$$
r=.80 \uparrow
$$

Patches: Non-colour-blind

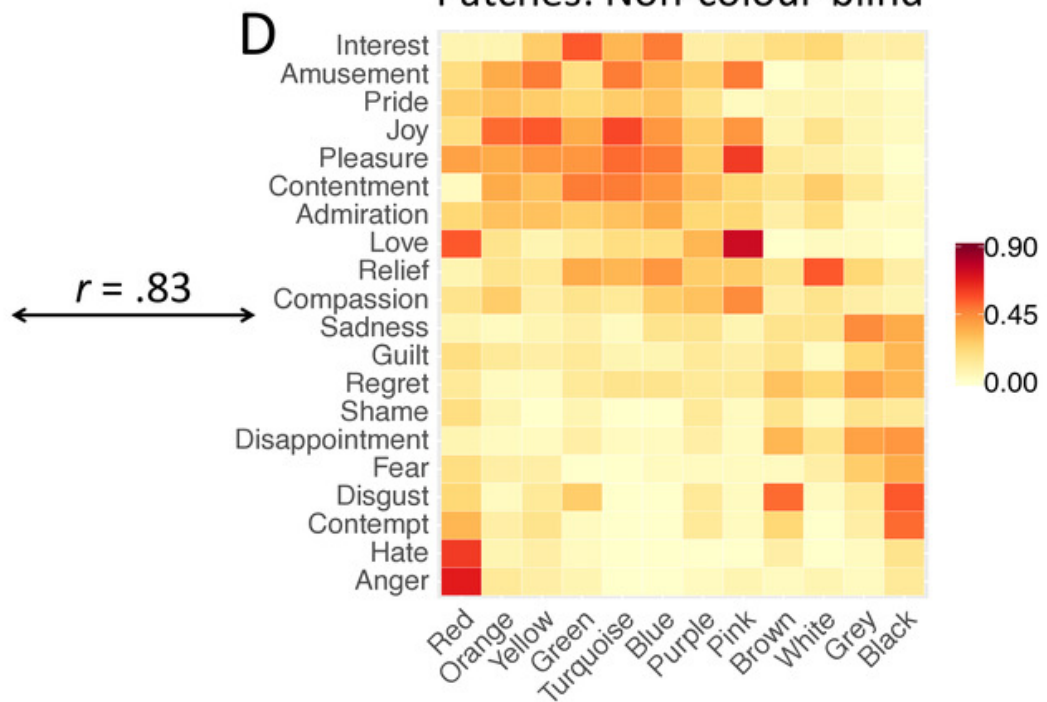




\section{Table $\mathbf{1}$ (on next page)}

Demographic information of colour-blind and non-colour-blind participants, divided by condition 
1 Table 1. Demographic information of the colour-blind and non-colour-blind participants, divided by 2 condition.

\begin{tabular}{|c|c|c|c|c|c|c|c|}
\hline & & \multirow[t]{2}{*}{$N$} & \multicolumn{2}{|l|}{ Age } & \multirow[t]{2}{*}{ Gender } & \multicolumn{2}{|c|}{ French fluency $(\max 8)$} \\
\hline & & & Mean & $S D$ & & Mean & $S D$ \\
\hline Colour terms & Colour blind & 30 & 24.93 & 4.46 & All males & 8 & 0.00 \\
\hline condition & $\begin{array}{l}\text { Non-colour- } \\
\text { blind }\end{array}$ & 31 & 23.55 & 3.38 & All males & 8 & 0.00 \\
\hline Colour & Colour blind & 34 & 22.56 & 5.71 & All males & 7.88 & 0.54 \\
\hline $\begin{array}{l}\text { patches } \\
\text { condition }\end{array}$ & $\begin{array}{l}\text { Non-colour- } \\
\text { blind }\end{array}$ & 34 & 23.53 & 3.95 & All males & 7.75 & 0.65 \\
\hline
\end{tabular}

3 


\section{Table 2 (on next page)}

Colour stimuli used in the terms and patches conditions.

Munsell values for colour patches taken from Lindsey \& Brown (2014). The last columns show the CIE1931 xyY values for our patches. 
1 Table 2. Colour stimuli used in the terms and patches conditions. Munsell values for colour patches taken

2 from Lindsey \& Brown (2014). The last columns show the CIE1931 xyY values for our patches.

\begin{tabular}{|c|c|c|c|c|c|c|}
\hline \multirow[t]{3}{*}{ Colour term } & \multicolumn{6}{|c|}{ Colour patch } \\
\hline & \multicolumn{3}{|c|}{ Munsell colour-order system } & \multicolumn{3}{|c|}{ CIE1931 coordinates } \\
\hline & Hue & Value & Chroma & $\mathrm{Y}\left(\mathrm{cd} / \mathrm{m}^{2}\right)$ & $x$ & $\mathrm{y}$ \\
\hline Red & $5.00 \mathrm{R}$ & 4 & 14 & 12.00 & .57 & .31 \\
\hline Orange & $5.00 \mathrm{YR}$ & 6 & 12 & 30.05 & .51 & .42 \\
\hline Yellow & $5.00 \mathrm{Y}$ & 8 & 14 & 59.44 & .45 & .48 \\
\hline Green & $2.50 \mathrm{G}$ & 5 & 12 & 20.99 & .27 & .50 \\
\hline Turquoise & $7.50 \mathrm{BG}$ & 6 & 8 & 30.38 & .22 & .33 \\
\hline Blue & $10.00 \mathrm{~B}$ & 6 & 10 & 30.05 & .20 & .24 \\
\hline Purple & $7.50 \mathrm{P}$ & 4 & 10 & 12.00 & .31 & .22 \\
\hline Pink & $7.50 \mathrm{RP}$ & 7 & 8 & 43.07 & .37 & .31 \\
\hline Brown & 7.50 YR & 3 & 6 & 6.55 & .49 & .42 \\
\hline White & $10.00 \mathrm{RP}$ & 9.5 & 0 & 90.01 & .31 & .33 \\
\hline Grey & $10.00 \mathrm{RP}$ & 6 & 0 & 30.05 & .31 & .33 \\
\hline Black & $10.00 \mathrm{RP}$ & 1.5 & 0 & 2.02 & .31 & .33 \\
\hline Grey (background) & $10.00 \mathrm{RP}$ & 5 & 0 & 18.58 & .31 & .32 \\
\hline
\end{tabular}

3

4 


\section{Table 3 (on next page)}

Matrix-to-matrix correlations per colour $\left(\mathrm{PSI}_{\text {colour }}\right)$, separated by correlations between colour-blind and non-colour-blind participant association matrices, and between colour terms and colour patches association matrices.

The $\mathrm{PSI}_{\text {colour }}$ (correlation coefficient $r$ ) indicates the similarity between two matrices with 1 indicating perfect similarity. All $p$-values are FDR corrected for multiple comparisons; $* p<$ $.050, * * p<.010, * * * p<.001$. 
1 Table 3. Matrix-to-matrix correlations per colour ( $\left.P\right|_{\text {colour }}$ ), separated by correlations between colour2 blind and non-colour-blind participant association matrices, and between colour terms and colour 3 patches association matrices. The PSI colour (correlation coefficient $r$ ) indicates the similarity between two 4 matrices with 1 indicating perfect similarity. All $p$-values are FDR corrected for multiple comparisons; ${ }^{*} p$ $5<.050, * * p<.010, * * * p<.001$.

\begin{tabular}{llllll}
\hline & \multicolumn{2}{l}{$\begin{array}{l}\text { Colour blind } \\
\text { colour-blind }\end{array}$} & & & \multicolumn{2}{l}{ Terms vs. Patches } \\
\cline { 2 - 3 } \cline { 5 - 6 } & Terms & Patches & & Colour-blind & $\begin{array}{l}\text { Non-colour- } \\
\text { blind }\end{array}$ \\
\hline Red & $0.88^{* * *}$ & $0.85^{* * *}$ & & $0.84^{* * *}$ & $0.82^{* * *}$ \\
Orange & $0.85^{* * *}$ & $0.77^{* * *}$ & & $0.83^{* * *}$ & $0.85^{* * *}$ \\
Yellow & $0.84^{* * *}$ & $0.90^{* * *}$ & & $0.83^{* * *}$ & $0.88^{* * *}$ \\
Green & $0.80^{* * *}$ & $0.55^{*}$ & & $0.46^{*}$ & $0.76^{* * *}$ \\
Turquoise & $0.83^{* * *}$ & $0.92^{* * *}$ & & $0.87^{* * *}$ & $0.95^{* * *}$ \\
Blue & $0.97^{* * *}$ & $0.86^{* * *}$ & & $0.84^{* * *}$ & $0.96^{* * *}$ \\
Purple & 0.07 & 0.09 & & 0.26 & $0.69^{* *}$ \\
Pink & $0.90^{* * *}$ & $0.87^{* * *}$ & & $0.89^{* * *}$ & $0.95^{* * *}$ \\
Brown & $0.79^{* * *}$ & $0.82^{* * *}$ & & $0.82^{* * *}$ & $0.84^{* * *}$ \\
Grey & $0.91^{* * *}$ & $0.76^{* * *}$ & & $0.86^{* * *}$ & $0.89^{* * *}$ \\
White & $0.60^{* *}$ & $0.88^{* * *}$ & & $0.75^{* * *}$ & $0.49^{*}$ \\
Black & $0.92^{* * *}$ & $0.86^{* * *}$ & & $0.67^{* *}$ & $0.68^{* *}$ \\
\hline
\end{tabular}

6 